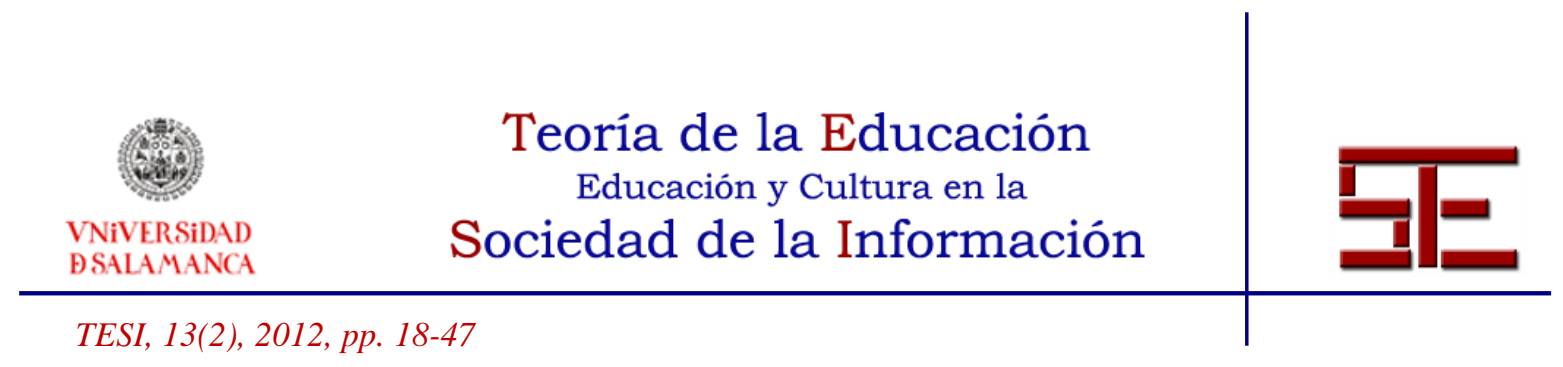

\title{
RECURSOS Y HERRAMIENTAS DIDÁCTICAS PARA EL APRENDIZAJE DE LA ROBÓTICA
}

Resumen: Este artículo analiza diferentes experiencias docentes que tienen como finalidad el aprendizaje de la robótica en el mundo universitario. Estas experiencias se plasman en el desarrollo de varios cursos y asignaturas sobre robótica que se imparten en la Universidad de Alicante. Para el desarrollo de estos cursos, los autores han empleado varias plataformas educativas, algunas de implementación propia, otras de libre distribución y código abierto. El objetivo de estos cursos es enseñar el diseño e implementación de soluciones robóticas a diversos problemas que van desde el control, programación y manipulación de brazos robots de ámbito industrial hasta la construcción y/o programación de mini-robots con carácter educativo. Por un lado, se emplean herramientas didácticas de última generación como simuladores y laboratorios virtuales que flexibilizan el uso de brazos robots y, por otro lado, se hace uso de competiciones y concursos para motivar al alumno haciendo que ponga en práctica las destrezas aprendidas, mediante la construcción y programación de mini-robots de bajo coste.

Palabras clave: robots educativos; robots de bajo coste; laboratorios virtuales y remotos; entornos interactivos; simuladores

Pablo Gil Vázquez, Carlos Alberto Jara, Santiago Timoteo Puente Méndez, Francisco Andrés Candelas Herías y Fernando Torres Medina 




\title{
EDUCATIONAL RESOURCES AND TOOLS FOR ROBOTIC LEARNING
}

\begin{abstract}
This paper discusses different teaching experiences which aims are the learning robotics in the university. These experiences are reflected in the development of several robotics courses and subjects at the University of Alicante. The authors have created various educational platforms or they have used tools of free distribution and open source for the implementation of these courses. The main objetive of these courses is to teach the design and implementation of robotic solutions to solve various problems not only such as the control, programming and handling of robot but also the assembly, building and programming of educational mini-robots. On the one hand, new teaching tools are used such as simulators and virtual labs which make flexible the learning of robot arms. On the other hand, competitions are used to motivate students because this way, the students put into action the skills learned through building and programming low-cost mini-robots.
\end{abstract}

Key words: educational robot; low-cost robots; virtual and remote labs; interactive environments; simulators

Pablo Gil Vázquez, Carlos Alberto Jara, Santiago

Timoteo Puente Méndez, Francisco Andrés Candelas Herías y Fernando Torres Medina 


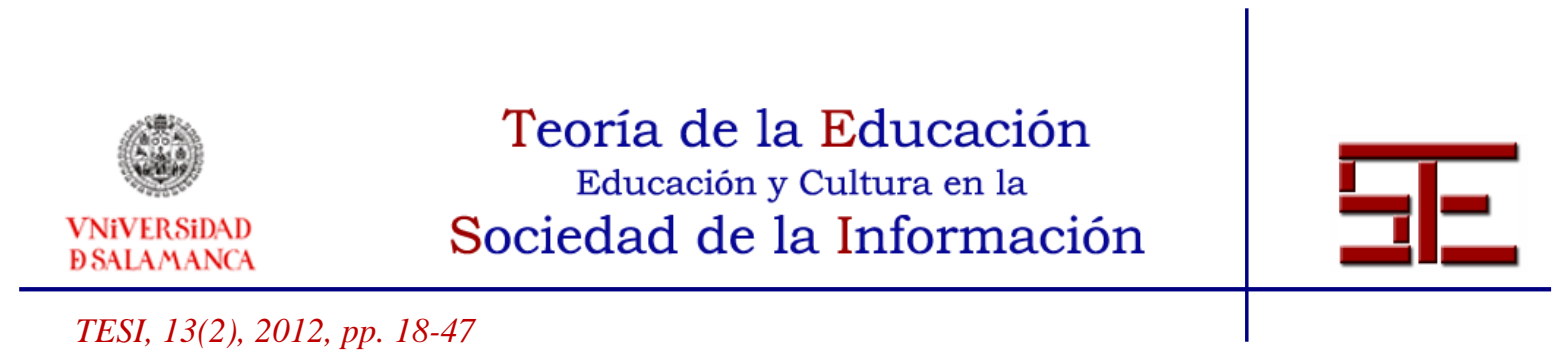

\section{RECURSOS Y HERRAMIENTAS DIDÁCTICAS PARA EL APRENDIZAJE DE LA ROBÒTICA}

Fecha de recepción: 25/05/2012; fecha de aceptación: 14/06/2012; fecha de publicación: 26 /07/2012

Pablo Gil Vázquez

pablo.gil@ua.es

Universidad de Alicante

Carlos Alberto Jara

carlos.jara@ua.es

Universidad de Alicante

Santiago Timoteo Puente Méndez

santiago.puente@ua.es

Universidad de Alicante

Francisco Andrés Candelas Herías

francisco.candelas@ua.es

Universidad de Alicante

Fernando Torres Medina

fernando.torres@ua.es

Universidad de Alicante

\section{1.- INTRODUCCIÓN}

En la actualidad, la industria nacional exige altos niveles de competitividad y productividad, al tiempo que se garantizan elevados estándares de calidad. La tecnología y en especial la robótica tienen una incidencia directa sobre el modelo productivo nacional y sobre el progreso y modernidad de un país. En los últimos años, los cursos de robótica están adquiriendo especial relevancia en los ambientes educativos. Es primordial que así sea, puesto que el futuro implica innovación tecnológica y ésta requiere, en muchos casos, que se disponga de conocimientos y destrezas en robótica y materias afines. Por este motivo, cada vez más, desde los sectores educativos se está haciendo el esfuerzo de proporcionar asignaturas y cursos que enseñen a nuestros alumnos tanto en las escuelas como en las universidades, a través de cursos de grado y postgrado, la asignatura de Robótica.

Pablo Gil Vázquez, Carlos Alberto Jara, Santiago

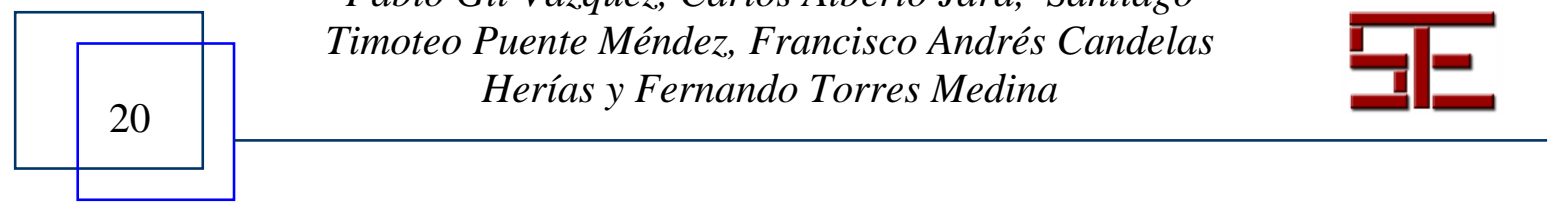




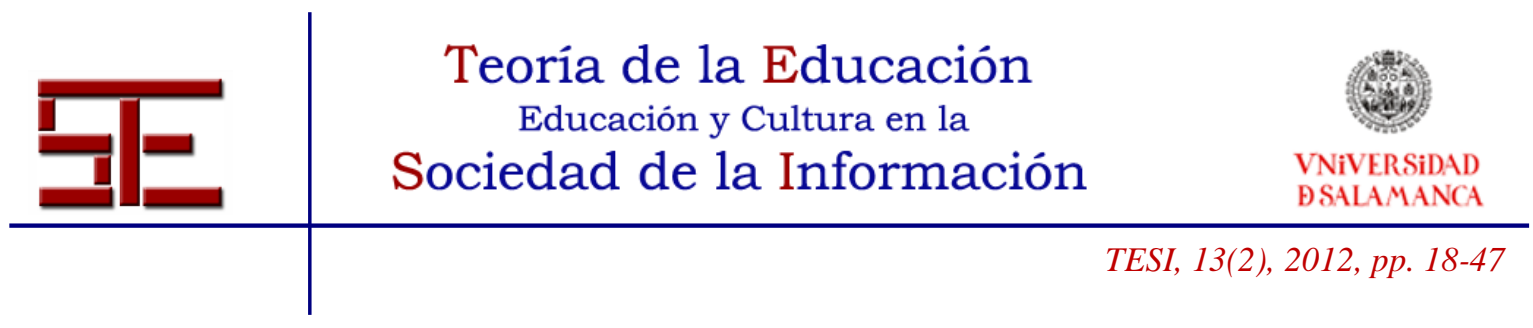

En este sentido, los cursos sobre robótica que se imparten en la Universidad de Alicante siempre han pretendido garantizar una formación técnica al tiempo que desarrollan una mentalidad innovadora entre los estudiantes. No sólo es importante aplicar soluciones robóticas a problemas conocidos en entornos industriales, sino que se busca que el alumno potencie y desarrolle nuevas ideas que puedan aplicar la robótica en campos emergentes como la asistencia o los servicios (CEA-GTrob, 2011).

Una buena educación en robótica implica tres aspectos básicos: interdisciplinaridad, constructivismo y colaboración. Así, por un lado, la enseñanza de la robótica requiere que se combine el aprendizaje del conocimiento en distintos campos como la electrónica, la ingeniería del software, la inteligencia artificial, la mecánica, etc. También, precisa ser constructivista, es decir, es muy motivador que el estudiante diseñe, implemente y ponga en funcionamiento su implementación, software y/o hardware, para observar su propia creación y cómo ésta resuelve el planteamiento de un problema concreto propuesto por el profesor. Y, por otro lado, la propia interdisciplinaridad que caracteriza la robótica y su carácter creativo e innovador requieren de un aprendizaje colaborativo. Así, en muchos cursos de robótica es común organizar a los estudiantes en grupos para que trabajen aunando esfuerzos en aras de un objetivo común. De este modo, el grupo incrementa destrezas al tiempo que se puede realizar una especialización de tareas para después integrarlas en un objetivo común, por ejemplo la construcción y programación de un mini-robot de bajo coste.

La educación en robótica en la Universidad de Alicante implica, básicamente, dos tipos de actividades o cursos. Por un lado, aquellos que enseñan robótica mediante la construcción y posterior programación de mini-robots de bajo coste. Por otro lado, aquellos otros cursos que enseñan robótica mediante el estudio cinemático y dinámico del comportamiento de robots comerciales de ámbito industrial y su posterior programación para el desarrollo de tareas específicas de manipulado. A lo largo de este artículo, se comentarán ambos.

Este artículo, describe la experiencia de los autores en la elaboración de cursos de robótica en el ámbito educativo universitario y se organiza de la siguiente manera: en esta primera sección se hace una descripción de las herramientas hardware y software más empleadas en la robótica educativa. En la sección 2, se comentan las características de las herramientas que se usan en la Universidad de Alicante para la enseñanza de los cursos y asignaturas de robótica, algunas de ellas implementadas y desarrolladas por los autores del artículo. Éstas se caracterizan por ser herramientas libres, abiertas, gratuitas

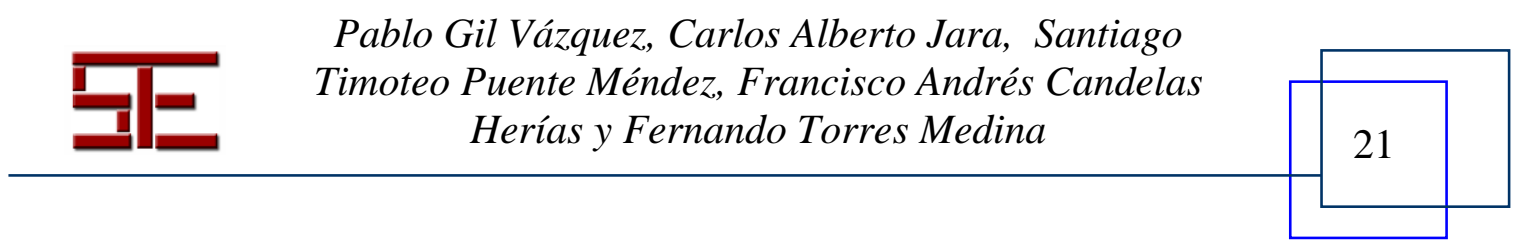




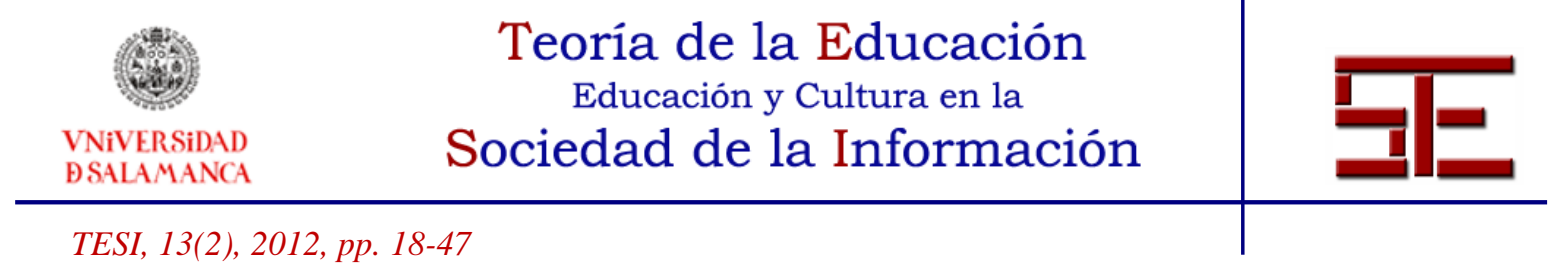

y/o de bajo coste. En la sección 3, se detallan qué cursos de robótica se imparten, cuál es su metodología y cómo se organizan éstos. Finalmente, en las secciones 4 y 5 se analiza la opinión de los estudiantes que han cursado o han participado en estos cursos durante estos últimos años y se detallan las conclusiones extraídas de la experiencia docente en robótica con este tipo de herramientas.

\section{1.- Robots educativos de bajo coste: móviles y humanoides}

Los cursos de construcción y programación de robots requieren de plataformas robóticas que se caractericen por varios aspectos: bajo-coste, montaje sencillo e intuitivo, fácil programación, capaz de moverse de manera autónoma sin estar conectado por cable a un ordenador, flexible y con la capacidad de equiparlo por ejemplo de sistemas de percepción o sensores. Dentro de este grupo de plataformas robóticas educativas las más comunes son los robots móviles inteligentes y los robots humanoides. En los cursos que en este artículo se presentan han sido empleados un robot móvil Skybot (Skbybot, 2008) y un robot humanoide Robonova (Robonova, 2005). No obstante, en el mercado existe una gran diversidad de kits y materiales para la construcción de robots de alguno de estos tipos. Además del humanoide Robonova de la empresa HiTec destacan otros kits como el robot humanoide Biolid (Biolid, 2010) de la empresa Robotis, que destaca por su modularidad, flexibilidad y escalabilidad o el robot DARrwIn-Op, (Darwin, 2011) que está considerado el robot humanoide educativo más avanzado tecnológicamente del mercado actual.

Desde un punto de vista meramente educativo cabe destacar principalmente dos categorías de kits robóticos: kits comerciales (Nourbakhsh, 2005) y kits personalizados. Como kits comerciales se pueden agrupar todos aquellos fabricantes que proporcionan todas las piezas necesarias para la construcción de un robot facilitando el montaje e integración de componentes bajo la garantía de una única marca y de manuales bien documentados. En este grupo los más populares son los kits comerciales: VEX (Vex, 2007), K-Team (K-Team, 1999) y LEGO Mindstroms (LEGO, 1998). Es más, el $90 \%$ de los cursos de construcción y programación de robots emplean plataformas robóticas LEGO por su flexibilidad y por su software para la programación (Vavassori, 2012).

Todos estos fabricantes de kits robóticos destacan porque sus robots permiten emplearlos como herramientas en el aprendizaje de robótica básica y/o avanzada. Así se pueden elaborar cursos de programación en tiempo real, detección de obstáculos, navegación y aprendizaje, control automático, comunicaciones para gestionar redes de robots que trabajan cooperativamente, etc. El principal inconveniente de los kits

Pablo Gil Vázquez, Carlos Alberto Jara, Santiago

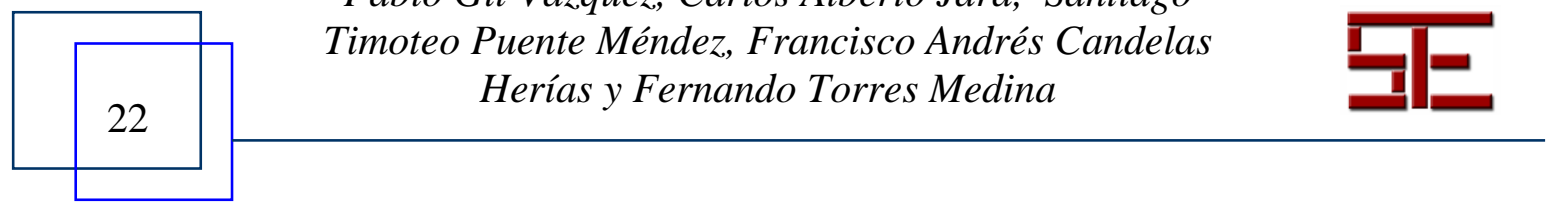




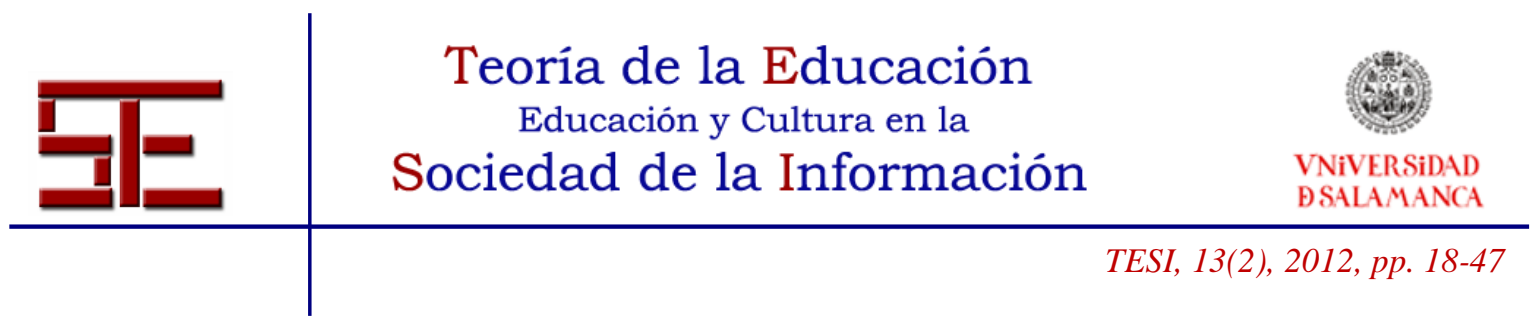

comerciales es que son caros y en muchos casos se ocultan algunos detalles del diseño puesto que no proporcionan sistemas totalmente abiertos desde un punto de vista de programar cualquier dispositivo de los que integren.

Los kits personalizables son aquellos cuyos componentes proceden de diferentes fabricantes y se adquieren por separado. Esto implica que un curso de construcción de un robot no se reduce a un mero ensamblado de piezas y componentes de acuerdo a un manual. Por este motivo, con este tipo de kits los estudiantes adquieren más conocimientos y habilidades en mecatrónica que con un kit comercial. Por lo general, destacan porque son mucho más económicos, el único coste es la compra de cada uno de los componentes necesarios que se emplearán para su construcción. Además, tienen la ventaja de que es posible manipular y programar cada componente a muy bajo nivel. Algunos de los kits robóticos personalizables más populares son: Onubot (Cañada, 2011), capaz de reconocer y seguir líneas; EmbedIT (Assaf, 2011), que destaca por disponer de módulos hardware intercambiables que permiten configurar distintos tipos de robots como cuadrúpedos, bípedos, etc. Trikebot (Nourbakhsh, 2005), que permite además de seguir líneas seguir colores mediante una webcam; eSwarBot (Couceiro, 2011), o el propio Skybot (Skybot, 2008), que se ha escogido por parte de los autores.

Con cualquiera de estos dos tipos de plataformas los estudiantes pueden fácilmente adquirir destrezas de programación y adquirir conocimiento en tareas propias de robótica como son: la planificación de movimientos para realizar una tarea, control de cada uno de estos movimientos, sensorizado y detección de objetos del entorno para llevar a cabo con éxito esta tarea y/o la navegación y gestión de trayectorias con autonomía.

Para acercar la robótica el público en general y para atraer estudiantes que se interesen por formarse en técnicas y campos robóticos en la última década se han organizado desde diferentes organizaciones concursos y competiciones de robots educativos (Balogh, 2005) tanto móviles como humanoides. Entre ellos cabe mencionar a nivel internacional las competiciones que promueve desde 1992 la AAAI (American Association for Artificial Intelligence) (Blach, 2002) donde se evalúan la navegación autónoma de robots, la manipulación robótica, la interacción hombre-robot o por ejemplo los robots de servicio aplicados a tareas de rescate. La Robocup (Robocup, 1996; Kitano, 1998), que en vigor desde 1997 ofrece competiciones de fútbol entre equipos de robots, además de competiciones de baile, en ella participan más de 500 equipos de robots de más de 40 países distintos, y las múltiples competiciones de LEGO

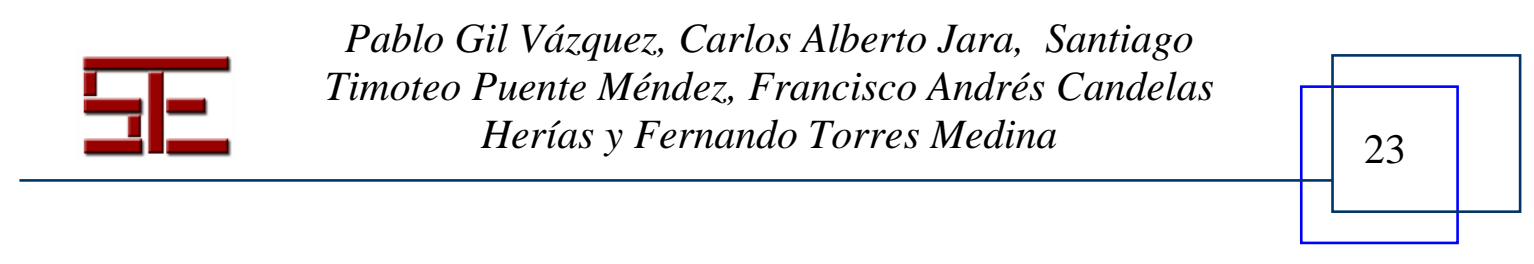






(Lego, 1999) extendidas internacionalmente en distintos niveles educativos. A nivel ibérico destacan en Portugal, Robótica (Robotica, 2001), que organiza diversas competiciones desde 2001 tales como fútbol, baile, salvamento o navegación, entre otras y Roboparty (Roboparty, 2012). En España desde 2001, Cybertech (Cybertech, 2001; Hernando, 2011), en la que se simulan robots que torean CEAbot (CEABot, 2006; Gonzalez-Fierro, 2010), donde se incluyen competiciones de lucha de robots humanoides o de navegación desde el 2006, y otras muchas como AESSBot (AessBot, 1994), Alcabot-Hispabot (Alcabot, 2000), CosmoBot (CosmoBot, 2008), Robolid (Robolid, 2003) o CampusBot (CampusBot, 2005), entre otras tantas.

También, desde la Universidad de Alicante se ha promovido la realización de este tipo de eventos como parte de la actividad formativa del estudiante (AUROBot, 2010). Con la realización de competiciones en el aula se pretende mejorar el trabajo en equipo entre estudiantes y potenciar las destrezas colaborativas (Murphy, 2001), al tiempo que se fomentan unas bases competitivas que motivan al estudiante para que mejore a base de creatividad e innovación, poniendo en práctica lo aprendido en su proceso de formación.

\section{2.- Simuladores de robótica y laboratorios virtuales-remotos}

En los últimos años, las aplicaciones telerrobóticas han crecido de forma rápida gracias al avance científico de las TIC. Nuevos tipos de tecnología, tales como la realidad virtual y la realidad aumentada, han mejorado la interacción del humano dentro del entorno virtual de la aplicación. Este hecho ha influenciado de manera significativa en el desarrollo de nuevos entornos dentro de mundo educativo para el aprendizaje a distancia. Además, el aumento de la fiabilidad y seguridad en Internet ha mejorado la calidad del proceso de teleoperación, mejorando las comunicaciones a través de la Red.

Tal y como se presenta en Dormido (2004) es posible distinguir cuatro tipologías de laboratorios en función del tipo de acceso local o remoto y del tipo de recurso físico al que se accede que puede ser real o simulado. Uno de los primeros laboratorios virtuales y remotos que introdujo el concepto de realidad virtual dentro de la interfaz de usuario fue el sistema desarrollado en la Universidad de Alicante, actualmente conocido como RobUALab.Ejs y en el que se ha estado trabajando durante más de una década (Torres, 1999; Puente, 2000; Candelas, 2003; Torres, 2006; Candelas, 2006; Jara, 2011a, 2011b). Este laboratorio cubre tres de las cuatro tipologías presentadas en Dormido (2004). Así permite teleoperar un brazo robótico Scorbot ER-IX y un Mitsubishi PA-10 a través de Internet con la ayuda de modelos virtuales 3D. Un usuario puede acceder a

Pablo Gil Vázquez, Carlos Alberto Jara, Santiago






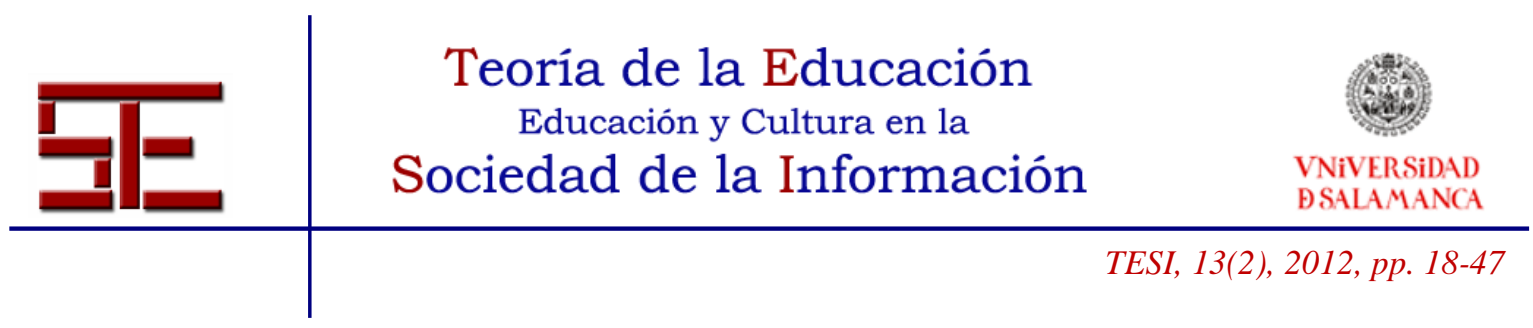

un applet Java. Para la simulación gráfica se emplean Java 3D y VRML, consiguiendo una interfaz de usuario muy realista. Además, este sistema incorpora la simulación del robot (modo off-line) para que el usuario pueda determinar si la ejecución de una serie de comandos es válida y no crea problemas en el robot remoto. Después de realizar una simulación y obtener una lista de comandos válidos, el usuario puede ejecutar la opción de teleoperación (modo on-line), solicitando al servidor web que se ejecuten los movimientos de la lista en el robot real.

Otro laboratorio virtual y remoto a destacar es el UJI Robot, capaz de controlar los movimientos de un robot vía web mediante comandos de alto nivel (Marín, 2005). Este sistema también incorpora un módulo de reconocimiento para el análisis y síntesis de voz y así poder ejecutar tareas de forma remota. Incorpora una interfaz con realidad aumentada para incrementar la sensación de telepresencia en el laboratorio remoto. Actualmente, es uno de los sistemas más completos. También cabe mencionar el laboratorio virtual y remoto presentado en Tzafestas (2006). Se trata de un sistema para la teleoperación a través de Internet de un robot Scara AdeptOne-MV. En este sistema la comunicación cliente-servidor está basada en el protocolo TCP/IP y la realimentación visual en el protocolo RTP. El applet cliente posee una representación virtual realizada en Java 3D del laboratorio remoto y el servidor es el encargado de enviar por el puerto serie los comandos recibidos desde el applet cliente.

Existen otros tantos, laboratorios virtuales y remotos en el mundo educativo, de características 'open-free'. Entre ellos cabe destacar a Cosma (2003), un laboratorio virtual y remoto utilizado en la docencia de robótica en Verona (Italia) con la posibilidad de teleoperar un robot industrial PUMA 560 y un robot móvil Nomad200. También es importante nombrar a Alencastre (2003), un entorno multiusuario creado por una interfaz realizada en Java con la que es posible teleoperar a diferentes plataformas robóticas (robots móviles e industriales) desde cualquier ordenador conectado a Internet. Finalmente, en Safaric (2005), se describe una herramienta de teleoperación a través de Internet de un robot industrial de 5 GDL, cuya interfaz virtual está construida con tecnología Java y VRML. La aplicación permite a los usuarios introducir trayectorias articulares para ser ejecutadas por el robot remoto y la herramienta comprueba si existe algún tipo de colisión entre el robot y el entorno.

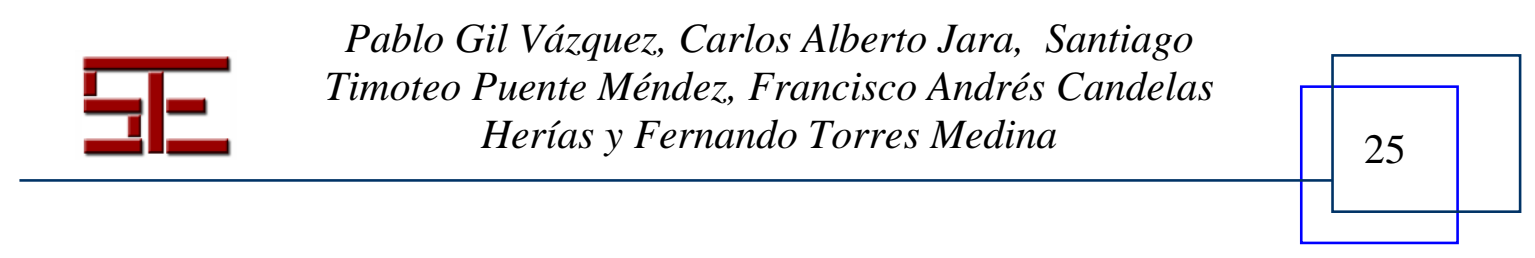




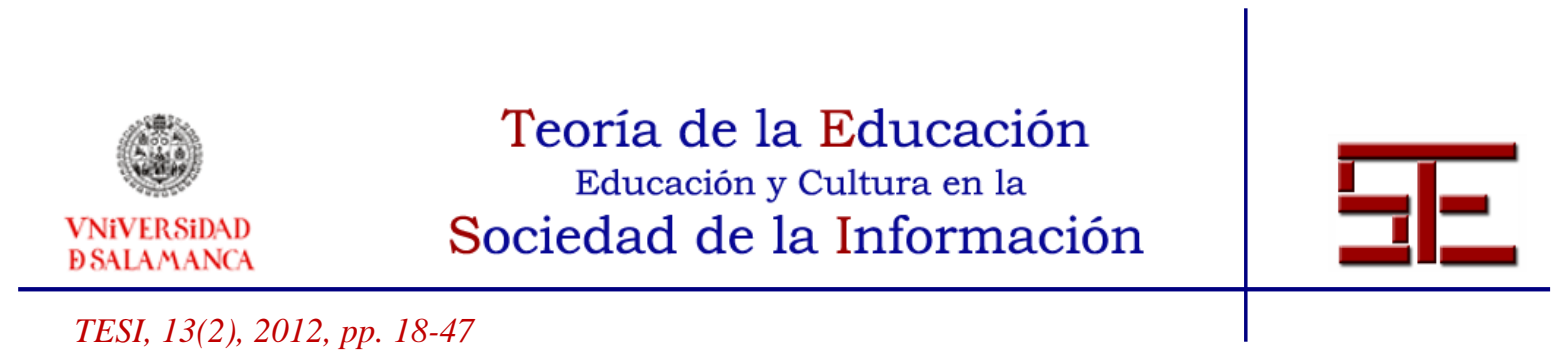

\section{2.- HERRAMIENTAS PARA EL APRENDIZAJE DE LA ROBÓTICA}

\section{1.- La construcción y programación de robots de bajo coste en la UA}

Actividades experimentales como la construcción y programación de un robot de bajo coste garantizan un aprendizaje interdisciplinar de la robótica. De este modo, el estudiante aprende robótica como una combinación de conocimiento de distintos campos como la electrónica, la ingeniería de software o la inteligencia artificial, que le van a permitir solucionar problemas. Además, es constructivista porque el estudiante desarrolla sus propios dispositivos y éstos interactúan con el mundo para llevar a cabo una tarea definida.

Los autores han elegido la plataforma móvil robótica Skybot (Skybot, 2012) que han modificado convenientemente para emplearla como base y herramienta fundamental para desarrollar un curso de construcción y programación de robots (AUROBot, 2010). De esta manera, los estudiantes ensamblan las piezas para construir su propio robot y lo programan para determinar su comportamiento y cómo éste interactúa con el entorno en el que se mueve (Puente, 2012). Además, como parte de la evaluación del curso, los robots de los estudiantes son sometidos a diferentes pruebas en un concurso o competición donde se demuestra qué capacidades tienen éstos para moverse de manera autónoma. Este tipo de organización hace que se garanticen los estados básicos de desarrollo de cualquier proceso de ingeniería: análisis de requerimientos (necesidades que debe cubrir el robot para llevar a cabo una tarea), diseño e implementación del prototipo (construcción y programación del robot) y verificación y rediseño de éste (durante el proceso de competición).

El robot Skybot (Figura 1) es una plataforma robótica simple, económica, educativa y de código abierto. Este tipo de robot se puede emplear para el aprendizaje de la robótica tanto en ambientes de educación secundaria como de grado o licenciatura. Además, como es un robot abierto, tanto los esquemas electrónicos del hardware como el código fuente para programarlo están disponibles sin costo. Esto permite mayor flexibilidad si se desean hacer cambios, ampliaciones o modificaciones sobre las piezas básicas que proporciona el kit.

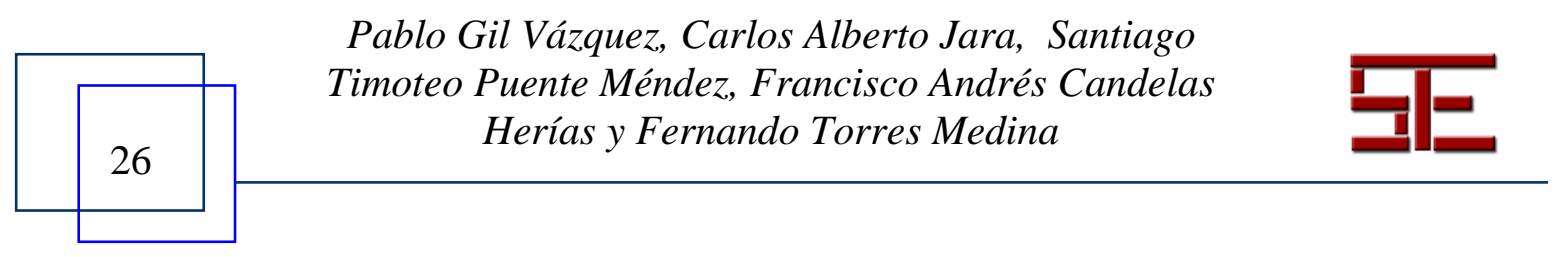



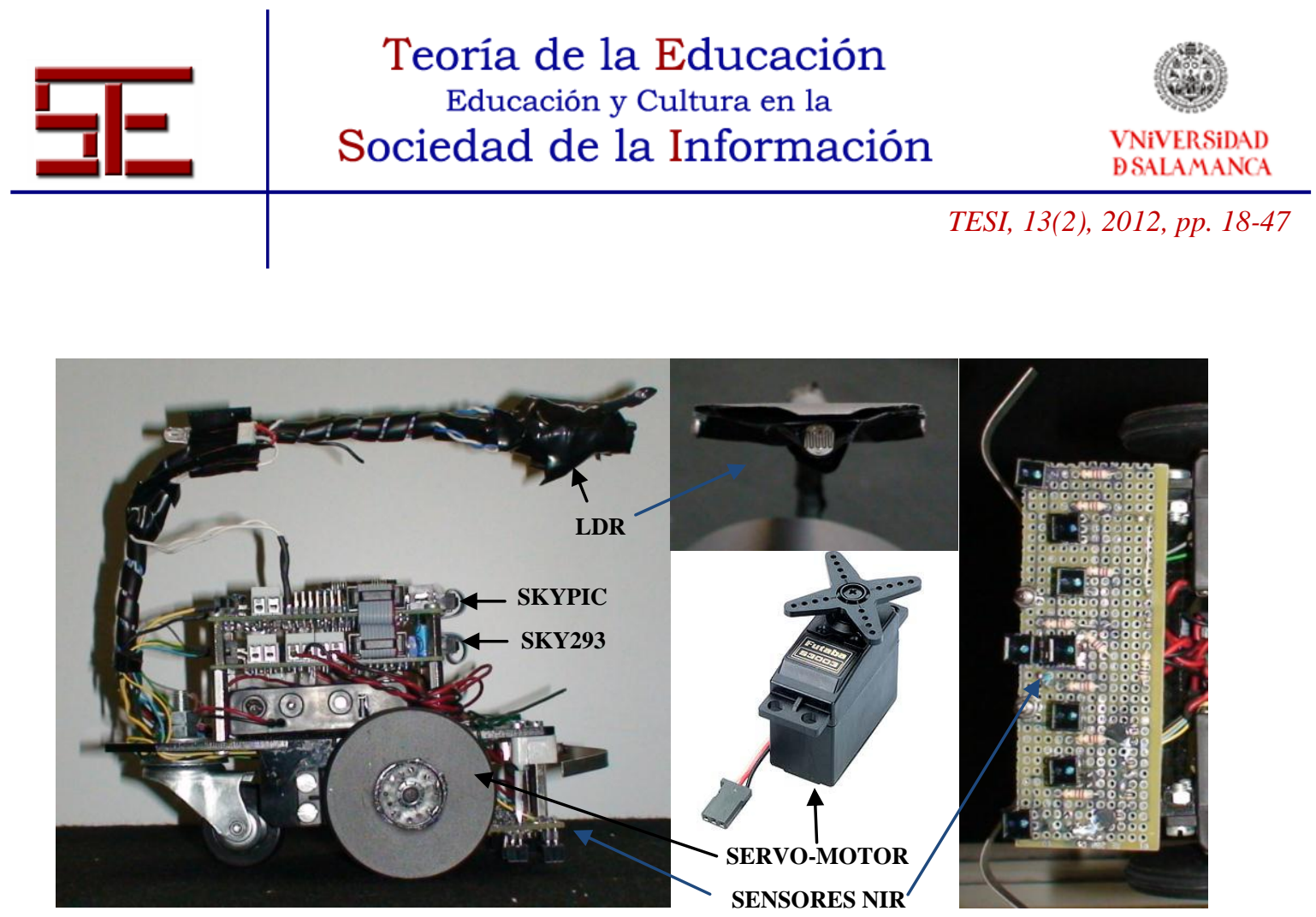

Figura 1. Modelo de Skybot ensamblado y programado en la Universidad de Alicante.

El robot Skybot (Skybot, 2008) que se ha empleado como parte de los cursos de construcción de robots en la Universidad Alicante consta de los siguientes componentes básicos: microcontrolador PIC 16F876A, circuito impreso SKY293 (PCB: Printed Circuit Board), dos mini-servomotores Futaba 3003, cuatro sensores infrarrojos CNY70 (NIR: Near Infra-red), sensor de contacto y un sensor de detección de luz (LDR: Light dependent Resistor). Su coste aproximado es de $175 €$ por kit.

El microcontrolador, SKYPIC-PIC 16F876A, está integrado en un chip y a través de su programación el estudiante puede ejecutar programas que controlen dispositivos externos. Unidades como un microprocesador, unidad de memoria, contadores, relojes, puertos de entrada/salida analógica o digital, puertos de comunicaciones, unidad de alimentación, etc., van integradas dentro del microprocesador, reduciendo el consumo y haciendo que todo sea fácilmente reemplazable. El circuito impreso, SKY293, tiene los interfaces necesarios para controlar los mini-motores y los mini-sensores. Este circuito impreso requiere cablearse para conectar el microcontrolador con esos otros componentes.

La programación del robot se reduce a programar el microcontrolador. Para programarlo se ha escogido el compilador SDCC. SDCC es un un programa abierto y libre distribuido con licencia GPL que proporciona un entorno sencillo para que el estudiante pueda crear sus propios programas en $\mathrm{C}$ (alto nivel) o en código ensamblador (bajo nivel) y este compilador genere el fichero hex con código máquina entendible por el micro-controlador. Los estudiantes de ingeniería de las titulaciones de

Pablo Gil Vázquez, Carlos Alberto Jara, Santiago

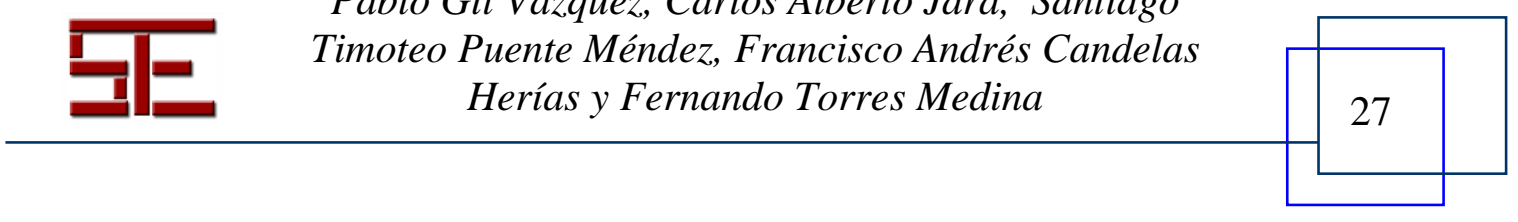




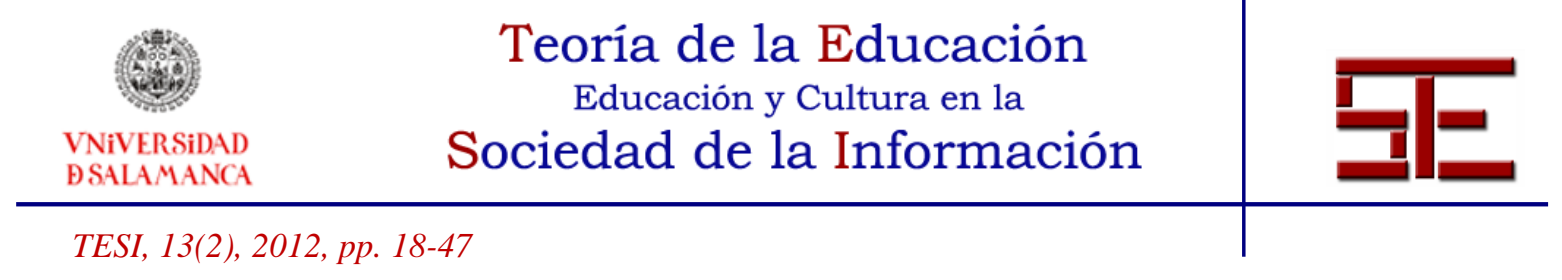

Telecomunicaciones e Informática que se imparten en la Universidad de Alicante en su mayoría tienen conocimientos previos de lenguaje $\mathrm{C}$. No obstante, en este tipo de cursos se recomienda al estudiante programar a bajo nivel porque la familia $16 \mathrm{~F}$ del PIC debido a la limitación de memoria para cargar programas. Esto implica, que el estudiante tiene que ser capaz de programar con estructuras de datos sencillas que optimicen los recursos físicos como la memoria del PIC.

Finalmente, los estudiantes usan Pydownloader como herramienta software con entorno gráfico para cargar el fichero .hex con el código máquina generado en la memoria del microcontrolador. Empleando estas herramientas software, el proceso para compilar y cargar un programa creado en $\mathrm{C}$ es muy simple.

Los autores también han empleado la plataforma humanoide Robonova (Robonova, 2005) para el aprendizaje en la programación de tareas de comportamiento y navegación en un entorno (Figura 2a).

\section{2.- El laboratorio virtual-remoto de la UA: RobUALab.Ejs}

RobUALab.Ejs es un laboratorio virtual y remoto desarrollado especialmente para la docencia y enseñanza de robótica industrial por el grupo de investigación en Automática, Robótica y Visión Artificial (AUROVA) de la Universidad de Alicante. La Figura $2 \mathrm{~b}$ muestra la planta real situada en un laboratorio de la universidad. Los dispositivos comerciales que componen la planta son un brazo robot tipo Scorbot ER-IX (Intelitek) de cinco grados de libertad, un pequeño almacén de piezas, una cinta transportadora y una mesa giratoria. De este modo, el equipo cliente envía un conjunto de trayectorias $\mathrm{u}$ órdenes de alto nivel al sistema robótico real, que han sido previamente simuladas y validadas en un entorno virtual que modela exactamente la planta remota. Posteriormente, el cliente visualiza el resultado de la teleoperación mientras se ejecutan las trayectorias mediante realimentación gráfica o de vídeo.

Pablo Gil Vázquez, Carlos Alberto Jara, Santiago

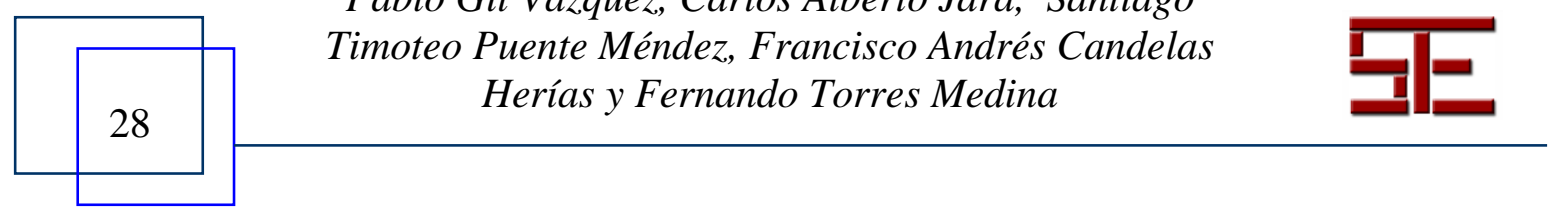



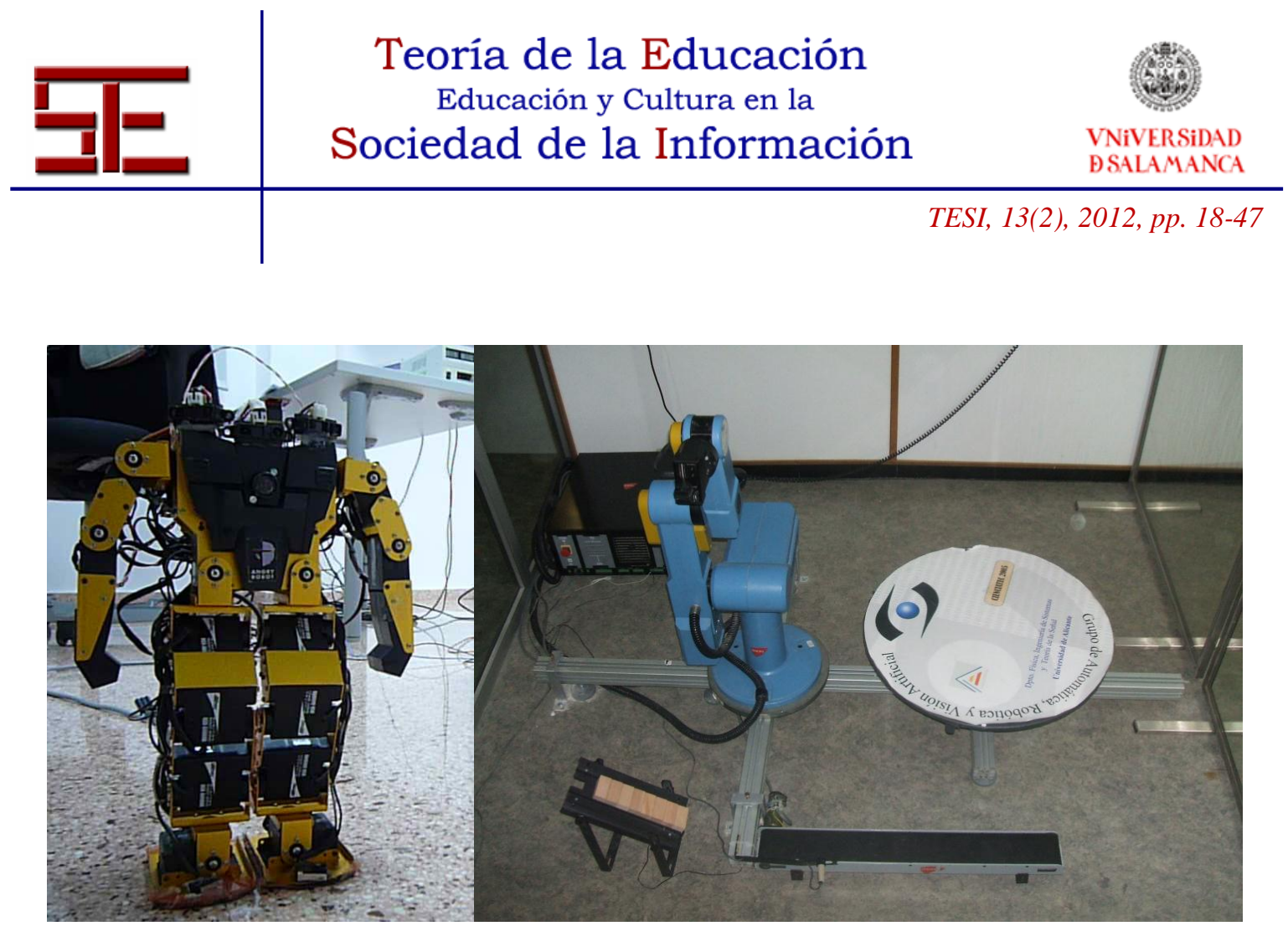

Figura 2. a) Robonova. b) Equipos reales del sistema RobUAlab.Ejs.

RobUAlab.Ejs es uno de los laboratorios incluidos en el proyecto AutomatL@bs (Vargas, 2010), del que forman parte varias universidades españolas. Para realizar prácticas con RobUAlab.Ejs como alumno es necesario acceder al entorno colaborativo que proporciona AutomatL@bs desde su portal web (http://lab.dia.uned.es/automatlab/). AutomatL@bs es una red de laboratorios virtuales/remotos para la enseñanza de la automática que se constituye mediante la integración de los recursos que aportan los grupos que participan en el proyecto. Proporciona un sistema de reserva de tiempos para la realización de los experimentos y un entorno de trabajo común que facilita su aprendizaje por parte del alumno.

El software cliente que proporciona la interfaz gráfica de usuario se puede descargar gratuitamente, y no es necesario que el usuario se registre para utilizar las funciones de simulación dentro del entorno virtual (http://robualab.eps.ua.es). Sin embargo, para poder usar las capacidades de operación remota, el usuario debe registrarse previamente como alumno en el proyecto AutomatL@bs, que se restringe actualmente a las universidades participantes en el proyecto.

\subsubsection{Arquitectura hardware y software del sistema RobUALab.Ejs}

Como muestra la Figura 3, el sistema se divide en dos partes principales conectadas a través de Internet: el equipo cliente que emplea un alumno y el conjunto de equipos que

Pablo Gil Vázquez, Carlos Alberto Jara, Santiago

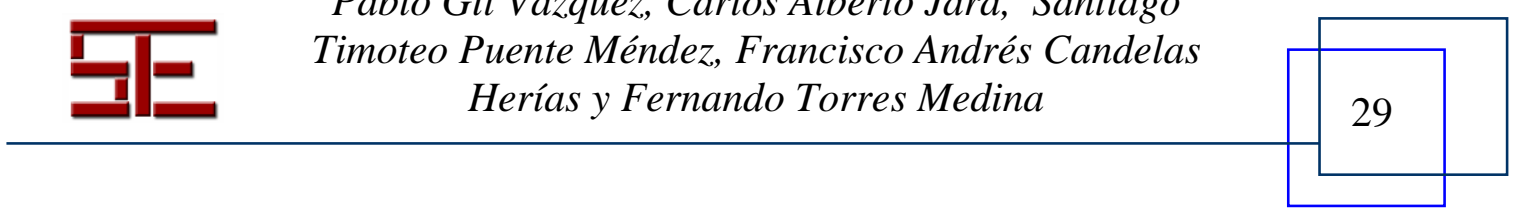






hay en el laboratorio de la universidad. La Figura 3 muestra los distintos componentes software empleados en cada equipo y los protocolos con los que se comunican entre sí.



Figura 3. Elementos que componen el sistema RobUAlab.Ejs.

El equipo cliente puede ser cualquier equipo con acceso a Internet y que disponga del siguiente software instalado: las bibliotecas de ejecución de Java y un navegador web con soporte de Java. El sistema operativo es indiferente, ya que la interfaz de usuario a la que accede el alumno es un applet Java que se carga en el navegador web. Sin embargo, sí que es necesario disponer de un equipo con un rendimiento de gráficos aceptable y un sistema operativo que soporte interfaz gráfica OpenGL. La experiencia del usuario será mejor si además dispone en su equipo de una tarjeta gráfica y de un sistema operativo con aceleración 3D. Todos estos requisitos son cumplidos habitualmente por los ordenadores personales actuales. Este applet ha sido creado utilizando la potente herramienta Easy Java Simulations (Ejs) (Esquembre, 2004), que facilita enormemente la creación de simulaciones basadas en Java. Con respecto al laboratorio real, se dispone de los siguientes equipos:

- Router-Firewall. Permite solo el tráfico a través de determinados puertos de TCP/IP, y realiza traducción de direcciones (NAT). Básicamente se permite el acceso a los puertos de los protocolos HTTP/HTTPS (portal web del sistema, descarga del applet cliente, acceso a la documentación, operación remota, etc.), cámara IP (también

Pablo Gil Vázquez, Carlos Alberto Jara, Santiago

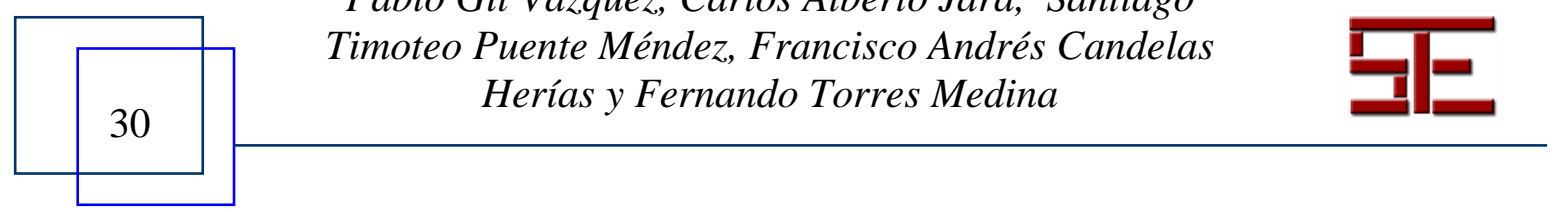




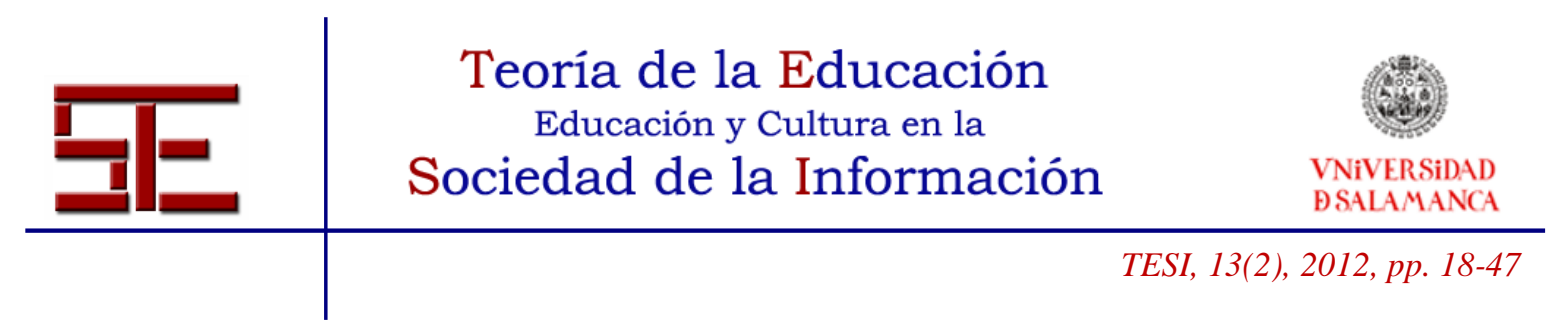

sobre HTTP, pero en otro puerto o dirección IP) y SSH (Secure Shell: para administración remota del Servidor Principal y del Servidor de Teleoperación).

- Servidor Principal. Este equipo es actualmente un servidor con sistema operativo Linux Suse, entre cuyo software destaca el servidor web Apache 2 con los módulos para SSL (Secure Socket Layer) y PHP, entre otros, el servidor de base de datos MySQL y las bibliotecas JRE de Java. Principalmente, las funciones de este equipo son la gestión de los usuarios, control de alimentación del laboratorio mediante un PLC, proporcionar los servicios web al cliente y comunicarse mediante el servidor de teleoperación.

- PLC. En el laboratorio se dispone de un armario con un PLC (Programable Logia Controller) S7-200 de Siemens y varios relés de potencia que controlan la alimentación de la iluminación de la zona de trabajo del robot y del propio controlador del robot, lo que permite activar esos dispositivos sólo cuando se utilizan. El PLC se comunica con un interfaz serie RS-232 con el Servidor Principal, y sobre este interfaz se ha implementado un sencillo protocolo basado en cadenas ASCII.

- Robot y su controlador. El robot empleado actualmente con RobUALab.Ejs es un brazo robot industrial modelo Scorbot ER-IX de la casa Intelitek, que dispone de cinco grados de libertad más una pinza, y soporta una carga máxima es de $2 \mathrm{~kg}$. El robot está conectado directamente al controlador que trae de fábrica, y este controlador se conecta con el equipo denominado Servidor de Tele-operación mediante un interfaz RS-322. Mediante este interfaz, se pueden enviar comandos al robot en lenguaje ACL como cadenas ASCII. A través de Internet el robot recibe comandos de alto nivel sobre las trayectorias a realizar.

- Servidor de Teleoperación. Este equipo es un elemento clave dentro del sistema RobUALab.Ejs, ya que se encarga de evitar un mal uso del robot que pueda dañarlo. Para ello, este equipo incluye un modelo virtual del entorno robot y su entorno, muy parecido al que ofrece el applet cliente al usuario final. Esta simulación se ejecuta también con Java3D y Java, en este caso sobre un sistema operativo Windows XP con soporte gráfico OpenGL. De este modo, los comandos con trayectorias de movimiento recibidos del Servidor Principal son simulados previamente a la ejecución real, y solo si no provocan velocidades excesivas o colisiones del robot con objetos del entorno o consigo mismo son finalmente enviados al controlador del robot. Para enviar los comandos al controlador del robot, estos son traducidos a cadenas ASCII que forman los comandos ACL correspondientes.

- Cámara IP. Se trata de una cámara comercial que incluye internamente un servidor web, y que es capaz de comprimir las imágenes y enviarlas por la Red mediante

Pablo Gil Vázquez, Carlos Alberto Jara, Santiago

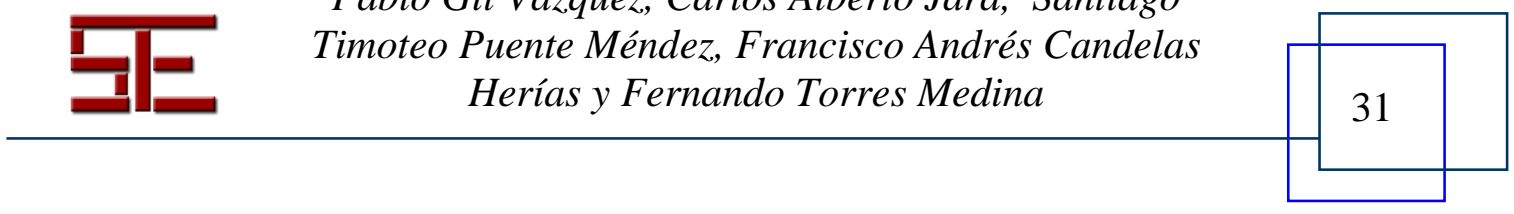




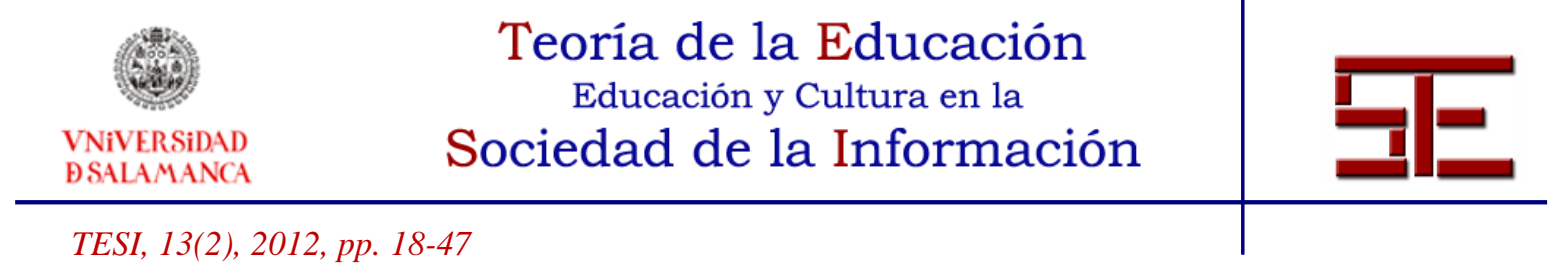

protocolos como HTTP. El applet cliente accede a las imágenes que proporciona esta cámara para mostrar una realimentación de vídeo o una visualización con realidad aumentada, mientras se está en modo de operación remota.

Como se ha mencionado, la comunicación a través de Internet entre el applet cliente y el servidor se realiza utilizando el protocolo HTTPS. Este protocolo, basado en el paradigma solicitud/respuesta, ha permitido simplificar las tareas de programación y configuración tanto del cliente como del servidor. De esta forma, el acceso al sistema remoto se realiza de manera similar al de una página web, evitando posibles problemas con bloqueos de puertos en firewalls. En el caso de los comandos de trayectorias enviados desde un cliente al Servidor Principal, los datos son codificados en cadenas URL e incluyen información referente al nombre de usuario, contraseña y las órdenes de alto nivel que deben ser ejecutadas en el robot real.

\subsubsection{La interfaz del laboratorio virtual}

La Figura 4 muestra la interfaz gráfica de usuario que proporciona el applet Java desarrollado mediante Ejs y su implementación 3D, que ha permitido diseñar un modelo muy realista de la planta remota. Más concretamente, la Figura 4 muestra el aspecto de la interfaz en el modo de simulación. Este modo proporciona el entorno virtual que permite una simulación completa de toda la funcionalidad de un robot real, incluyendo aspectos como la cinemática, dinámica, programación y planificación de trayectorias.

Entre las posibles opciones de simulación que ofrece el laboratorio virtual, destacan las descritas a continuación. Todas ellas son accesibles desde los botones que hay sobre la representación 3D del entorno virtual.

- Cinemática: Los alumnos pueden mover el robot especificando los valores angulares para las articulaciones del robot (cinemática directa) o las coordenadas cartesianas del efector en la articulación final (cinemática inversa). En cualquier caso, la aplicación resuelve la transformación cinemática necesaria y muestra los sistemas Denavit-Hartenberg sobre la simulación 3D, así como los valores de las matrices de transformación y Jacobiana en los paneles de datos mientras se realiza el movimiento.

- Planificación de trayectorias. Se pueden diseñar y simular movimientos avanzados mediante diferentes tipos de trayectorias articulares (síncrona, asíncrona, splines y polinómica 4-3-4) y cartesianas (lineal). Las trayectorias simuladas se pueden almacenar y concatenar en una Lista de Comandos, para ser ejecutadas secuencialmente.

Pablo Gil Vázquez, Carlos Alberto Jara, Santiago

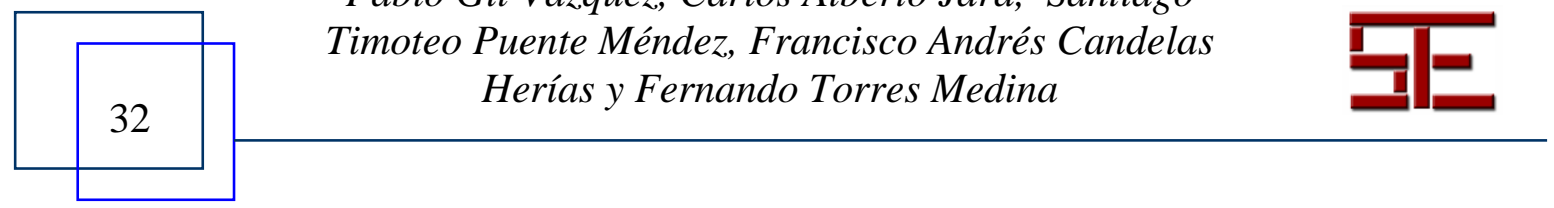




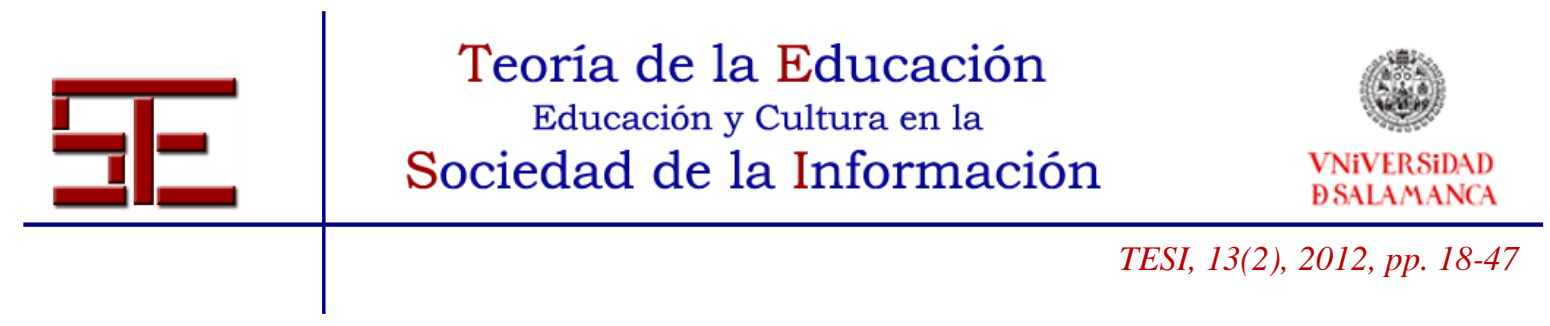

- Modelado de objetos. Los usuarios pueden especificar e introducir objetos virtuales en el entorno de simulación, para ser manipulados por el brazo robot para trasladarlos de sitio (por ejemplo, de la cinta transportadora a la mesa).

- Dinámica. Se pueden evaluar los pares desarrollados por los actuadores de las diferentes articulaciones mientras se simula una trayectoria. También, es posible modificar parámetros del modelo dinámico del robot como por ejemplo las masas e inercias de los eslabones y el peso de los objetos cogidos para ver su efecto en la simulación.

- Programación Off-line. Un alumno puede programar funciones en lenguaje Java para especificar tareas de manipulación más complejas. Para ello también hay disponibles diversas funciones y objetos que permiten manejar posiciones y trayectorias, así como ejecutar acciones de la pinza del robot, la cinta transportadora o la mesa. Un programa se puede compilar y después ejecutar en simulación.

- Cámara Virtual. La aplicación permite mostrar la vista de la proyección de una cámara en el extremo del robot (eye-in-hand camera), así como obtener imágenes de la misma para ser procesadas. De este modo es posible usar la aplicación para probar algoritmos de control visual (Jara, 2011a).

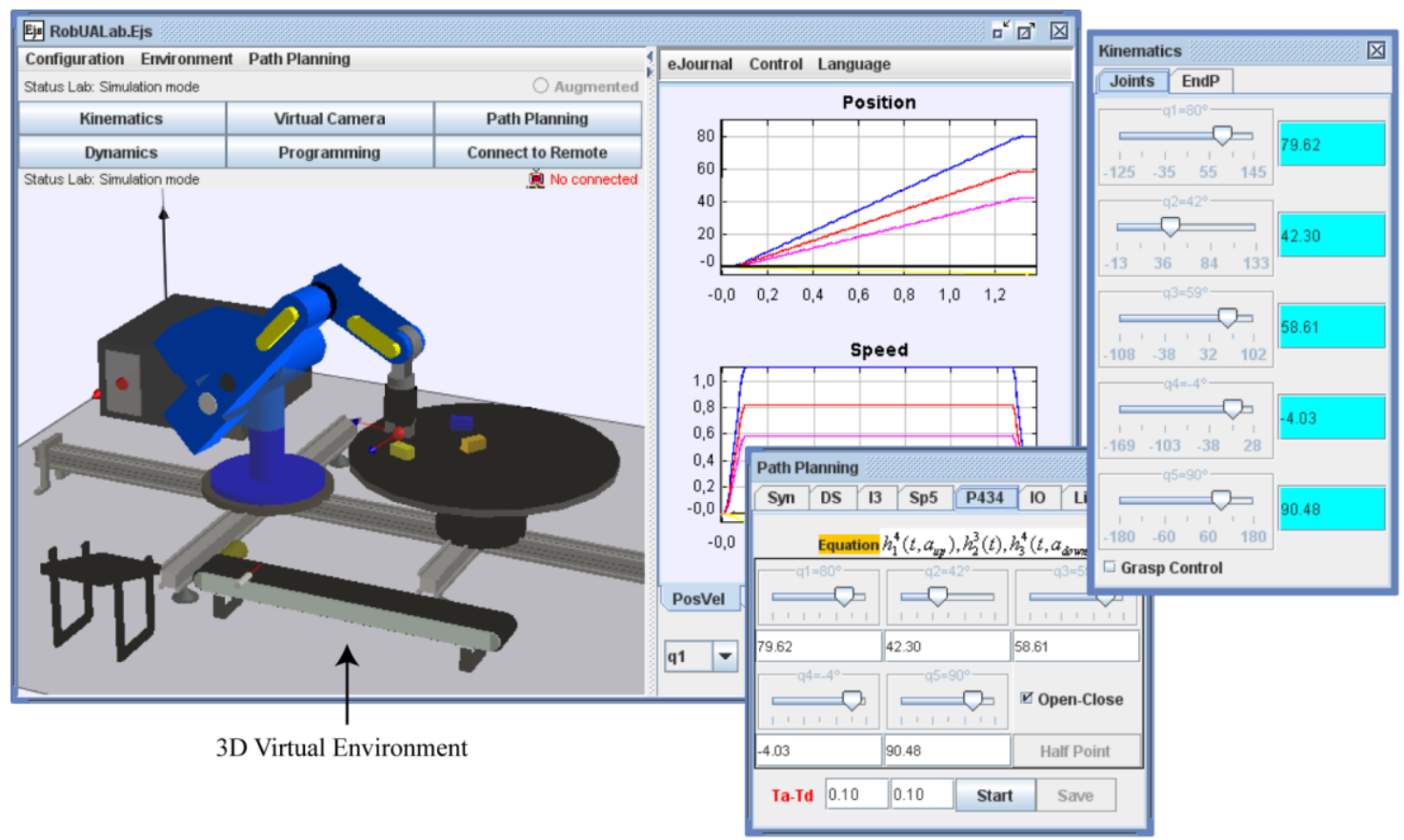

Figura 4. Interfaz de usuario proporcionada por el applet cliente de RobUALab.Ejs.

Pablo Gil Vázquez, Carlos Alberto Jara, Santiago

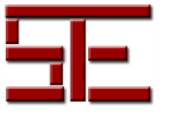

Timoteo Puente Méndez, Francisco Andrés Candelas Herías y Fernando Torres Medina 


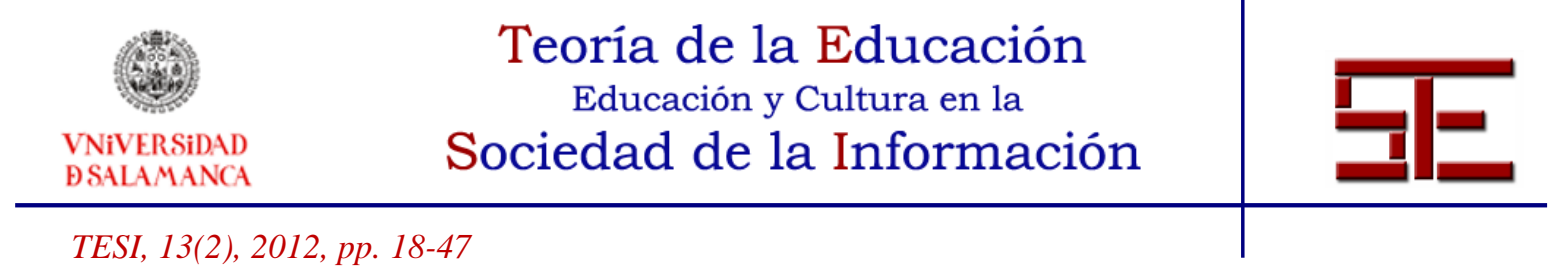

Cuando se activa el modo de operación remota pulsando el botón "Connect to remote", se solicita al usuario que se autentifique y especifique el Servidor de Teleoperación a utilizar. Si el usuario es validado correctamente por el Servidor Principal, y accede dentro una franja horaria que previamente ha reservado, entonces la interfaz de usuario pasa a modo de operación remota, y muestra una ventada con el flujo de vídeo recibido desde la cámara IP que hay en el laboratorio, según muestra la Figura 5. En el modo de operación remota se dispone de estas opciones:

- Control de la alimentación del robot y de la iluminación (solo usuarios habilitados), así como posibilidad de cambiar la perspectiva de la cámara IP.

- Control del robot real. La Lista de Comandos previamente generada en el modo de simulación puede ser enviada a los equipos del laboratorio para ejecutar las trayectorias en el robot real. Como se ha comentado en la sección anterior, tal lista de comandos será simulada de nuevo en el Servidor de Teleoperación antes de ser ejecutada en el robot real para prevenir colisiones y velocidades altas.

- Realimentación gráfica. Además de la realimentación de vídeo, la representación virtual 3D del robot se puede actualizar según las coordenadas del robot real mientras se mueve. Para ello, el Servidor de Teleoperación envía al applet cliente muestras de los valores de las articulaciones.

- Realidad aumentada (AR). Con esta opción, la vista principal es sustituida por la superposición entre objetos del mundo virtual 3D y las imágenes de vídeo del sistema real (Figura 5). Esta característica proporciona una información más clara al usuario, que facilita la comparación de la simulación con la ejecución real, y además es un factor que anima a los usuarios a experimentar con el sistema (Jara, 2011a).

Pablo Gil Vázquez, Carlos Alberto Jara, Santiago

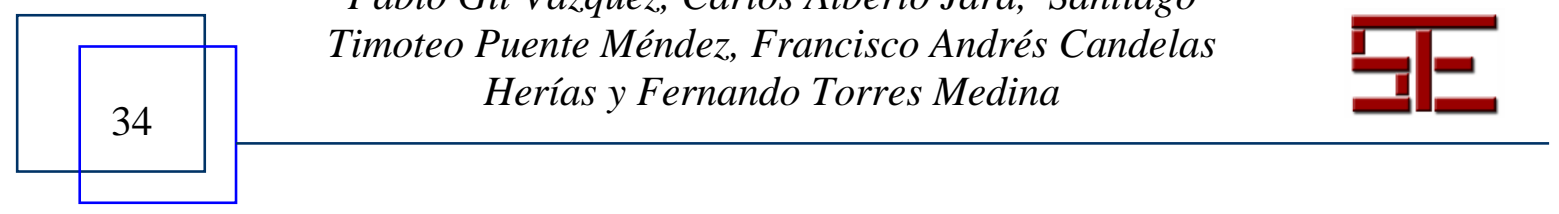



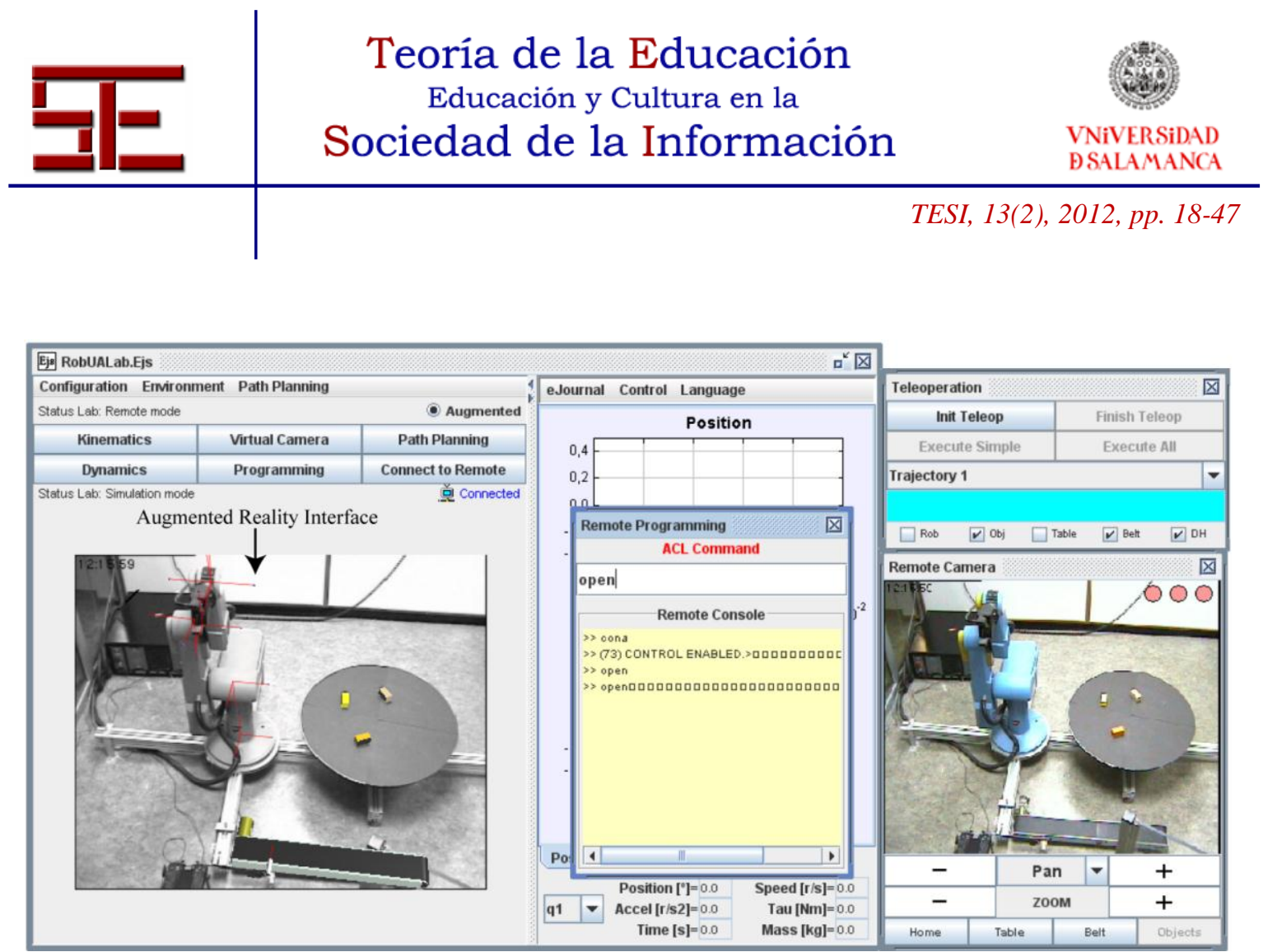

Figura 5. Aspecto de la interfaz de usuario del applet cliente en modo de operación remota con la opción de realidad aumentada (AR) activada.

\section{3.- EXPERIENCIA EDUCATIVA EN ROBÓTICA}

A continuación se describen los cursos que imparten los autores del artículo en el ámbito de la robótica dentro de los distintos estudios oficiales de la Universidad de Alicante. Los estudios oficiales abarcan tanto grados en ingeniería como cursos de postgrado (máster).

\section{1.- Robótica en el Grado de Ingeniería Informática y post-grado}

En las distintas titulaciones de informática como Ingeniero en Informática, Ingeniero Técnico en Informática de Sistemas e Ingeniero Técnico en Informática de Gestión se lleva impartiendo la asignatura "Robots y Sistemas Sensoriales" desde el curso 1998/99.

En la actualidad, la asignatura consiste en 60 horas distribuidas durante 1 cuatrimestre de sesiones de clase. Estas 60 horas se dividen en 30 horas teóricas y 30 horas prácticas de laboratorio. En teoría, se exponen los distintos componentes y subsistemas de un robot industrial, incluyendo el estudio cinemático y una introducción a la dinámica. Además, como contenidos de la parte teórica de la asignatura se realiza una introducción a los distintos tipos de sensores utilizados en robótica: tanto sensores internos de la propia estructura motriz del robot como sensores externos que proporcionan información de percepción del entorno. En la parte práctica de laboratorio

Pablo Gil Vázquez, Carlos Alberto Jara, Santiago

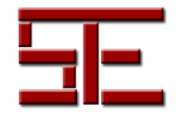
Timoteo Puente Méndez, Francisco Andrés Candelas Herías y Fernando Torres Medina 




se realizan experimentos de programación del robot Scorbot ER-IX (Figura 2b) mediante el laboratorio virtual RobUALab.Ejs (Figura 3), donde los alumnos se familiarizan con las características de un robot (Figura 4), así como con los requisitos necesarios para definir una tarea secuencial y repetitiva. Posteriormente, se plantea a los alumnos que realicen la misma experiencia pero programando el controlador real del robot. En este caso, el concepto práctico es el mismo, pero ya conocen las características del robot y su comportamiento, con lo que solo tienen que adaptar su programa al lenguaje de programación del robot. De este modo, los estudiantes aplican lo aprendido en el laboratorio virtual directamente sobre el controlador del robot sin interfaz gráfico amigable que simule comportamientos. El propio laboratorio virtual RobUALab.Ejs tiene diseñadas opciones para enviar comandos propios del lenguaje del robot, ACL, sobre el controlador para generar movimientos cartesianos y articulares (Figura 5).

Por un lado, para la realización de los experimentos con el simulador los estudiantes emplean 5 sesiones de prácticas, de 2 horas cada una, teniendo la opción de ampliar la práctica utilizando el simulador desde casa, aunque la mayoría destacan que prefieren las sesiones presenciales ya que les permiten solventar las dudas directamente con el profesor. Por otra parte, para la realización de la práctica con el robot real, se dedican también 5 sesiones de 2 horas cada una, a falta de probarla en el robot real, en este caso las prácticas eran totalmente presenciales, ya que el estudiante no puede probar la práctica si no es en el laboratorio con el robot real. El resto de horas de prácticas se dedican a ver otros conceptos en los que no se utilizan los laboratorios virtuales ni las herramientas presentadas en este artículo.

Hay que destacar que se ha realizado esta experiencia durante varios cursos académicos, dividiendo a los alumnos en dos grupos, unos que realizan primero la programación con el simulador y después la programación con el sistema real, y el otro grupo de alumnos al contrario. Se ha podido comprobar que el resultado obtenido en las prácticas, así como el tiempo invertido para su realización ha sido menor en el caso de los alumnos que han utilizado primero el simulador (ver Sección 4.2).

Pablo Gil Vázquez, Carlos Alberto Jara, Santiago






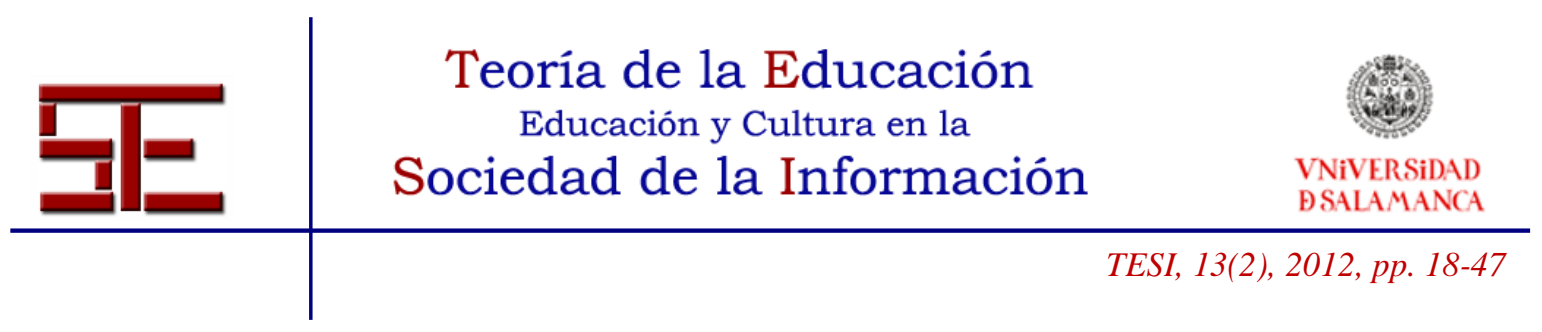

ASIGNATURAS

EN CURSOS DE GRADO Y MÁSTER

\begin{tabular}{|c|c|c|c|c|c|}
\hline Asignaturas & Grado & Post-grado & Horas $(\mathrm{T})$ & Horas $(\mathrm{P})$ & Tipo \\
\hline Robots y Sistemas Sensoriales & SÍ & NO & 30 & 30 & Optativa \\
\hline Robótica & NO & SÍ & 30 & 30 & Obligatoria \\
\hline $\begin{array}{l}\text { Nuevas Tendencias de la } \\
\text { Robótica }\end{array}$ & NO & SÍ & 15 & 15 & Optativa \\
\hline $\begin{array}{l}\text { Control y Programación de } \\
\text { Robots }\end{array}$ & NO & SÍ & 15 & 15 & Optativa \\
\hline
\end{tabular}

Figura 6. Tabla de distribución de asignaturas con temática de robótica.

En referencia a los estudios de post-grado, se imparten conceptos de robótica, principalmente, en las asignaturas "Robótica", "Nuevas tendencias de la robótica" y "Control y programación de robots", que se llevan impartiendo en el Máster Universitario en Automática y Robótica de la Universidad de Alicante desde su creación en el curso 2010/11 (Figura 6).

En la asignatura de "Robótica" se realiza una práctica con el simulador RobUALab.Ejs junto con la programación del robot real. A esta práctica se le asocia una sesión completa de 2 horas y media, el resto de dedicación en horas se emplea a formar al estudiante en otras herramientas y tecnologías fuera del ámbito de este artículo. En esta sesión se pretende que los estudiantes se familiaricen con un simulador de robótica, así como con un robot industrial. La práctica se enfoca, principalmente, partiendo de un esquema ya diseñado de la tarea a realizar. Los estudiantes tienen que completar el diseño e implementar la tarea con el robot. En este caso se ha preferido no realizar más prácticas con el simulador RobUALab.Ejs y conocer algunos simuladores comerciales para familiarizar a los estudiantes con software que pueden encontrarse después en el mundo laboral. Así, el software de simulación virtual se emplea para mostrar las ideas iniciales y los conceptos básicos.

En el caso de los estudios de post-grado, en la asignatura "Nuevas tendencias de la robótica", los estudiantes utilizan el robot Robonova-I (Figura 2a) para que practiquen con robots de bajo coste no industriales. Los experimentos que desarrollan se planifican en 4 sesiones de dos horas y media cada una, consiste en programar el robot humanoide para que ande por un entorno y sea capaz de esquivar los obstáculos que aparezcan en su entorno, las otras 5 horas se emplean para manejo de otras herramientas.

Pablo Gil Vázquez, Carlos Alberto Jara, Santiago

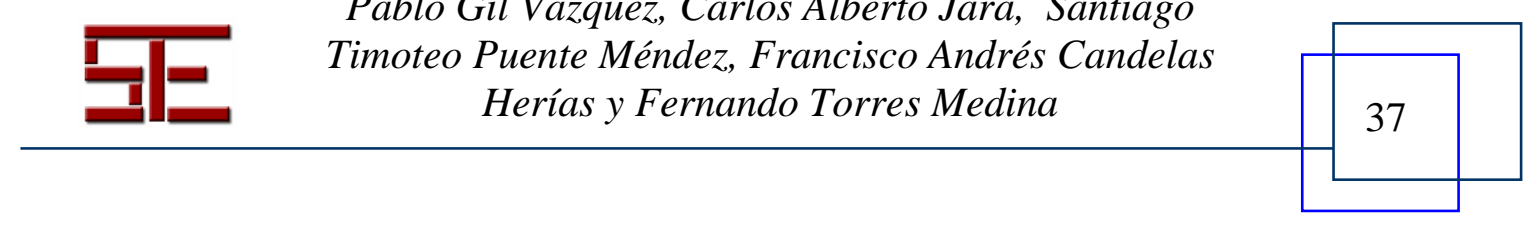




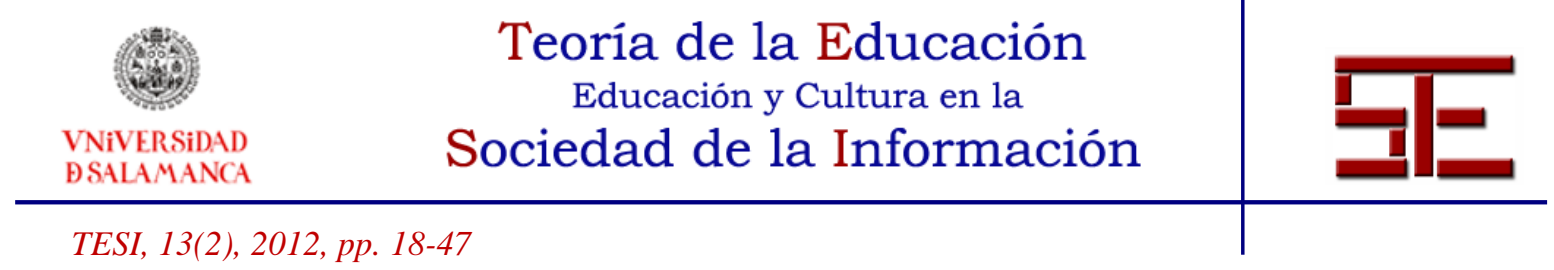

El robot Skybot (Figura 1), se utiliza para la docencia en la asignatura "Control y programación de robots", donde sirve como plataforma de pruebas para que los estudiantes implementen sus propios algoritmos de control para robótica móvil. Para ello, se ha añadido una conexión a entre el robot Skybot y una placa Arduino mediante el protocolo I2C, para facilitar la programación de estos robots por parte de los estudiantes.

\section{2.- Robótica como asignatura extracurricular}

Desde el 2010, los autores han diseñado varios cursos prácticos extracurriculares de introducción a la robótica. Estos cursos se han diseñado como talleres donde se busca que el estudiante construya y programe su propio robot desde cero. Este tipo de talleres está orientado a todo tipo de estudiantes con perfil ingenieril, bien sean estudiantes cursando estudios o que hayan finalizado éstos.

Los principales objetivos por los que se desarrollan este tipo de cursos son dos. Por un lado, introducir en las titulaciones materias robóticas que requieren un conocimiento transversal sobre todo en aquellas ingenierías donde la robótica no forma parte de los planes de estudio como asignatura troncal u obligatoria. Por otro lado, motivar al alumno en el aprendizaje de un campo novel en nuestro país empleando una metodología en la que se aprende jugando y experimentando.

Los detalles sobre estos talleres se pueden encontrar en AUROBot (2010). En la actualidad, el taller consiste en 50 horas distribuidas durante 1 semana de sesiones de clase. Estas 50 horas se dividen en 15 horas teóricas donde se exponen los principios básicos necesarios para la construcción y programación del robot y en 35 horas prácticas de laboratorio. Se suelen emplear 2 días para ensamblar todas las piezas del robot y cablear la electrónica y dispositivos que integra. Y los otros 3 días se emplean para, una vez comprobado que la construcción del robot es correcta, programar los algoritmos que determinarán su comportamiento para que éste pueda moverse de manera autónoma por un entorno o realizar alguna tarea concreta lo más inteligentemente posible.

En las horas teóricas se introducen y explican cuáles son los pasos que los estudiantes tienen que ir realizando durante cada sesión. Por ejemplo, si inicialmente el estudiante tiene que ensamblar un conjunto de piezas que implican servomotores y distintos circuitos impresos, el personal docente explica qué características tienen esos componentes, para qué se usen y cómo funcionan. De esta manera, el estudiante tiene

Pablo Gil Vázquez, Carlos Alberto Jara, Santiago






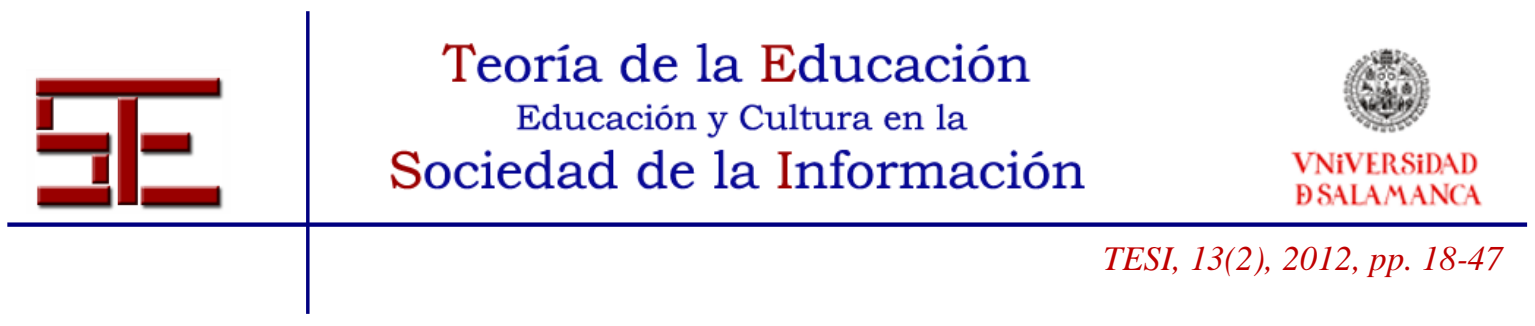

una visión general de la electrónica, mecánica y automática de los sistemas que compondrán el robot. Además, estas sesiones de teoría se acompañan de horas prácticas en la que los estudiantes ensamblan chasis, ruedas, sensores, circuitos, etc., para, posteriormente, cablear éstos y realizar las primeras pruebas de funcionamiento de motricidad del robot.

Una vez el robot ha sido construido, el resto de dedicación horaria se destina a la parte de programación. En estas sesiones, los docentes enseñan cómo se instala el software en un PC para programar el robot y se explica una introducción a microcontroladores que permita a los estudiantes conocer cómo funcionan, qué beneficios tienen y qué limitaciones. Posteriormente, se proporcionan las directrices generales para implementar un programa que haga uso de los sistemas sensoriales del robot: infrarrojos y resistores dependientes de la luz LDR, al tiempo que permita programar la odometría de éste para realizar alguna tarea, como por ejemplo realizar el seguimiento de líneas negras en un circuito.


Figura 7. Competiciones UA. a) Navegación y seguimiento. b) Combate para explosionar globos.

Para concluir el curso, los alumnos evalúan sus robots mediante pruebas competitivas en un concurso (Figura 7). La competición es una buena manera de testear el robot construido y medir el comportamiento y destrezas adquiridas para solucionar problemas

Pablo Gil Vázquez, Carlos Alberto Jara, Santiago

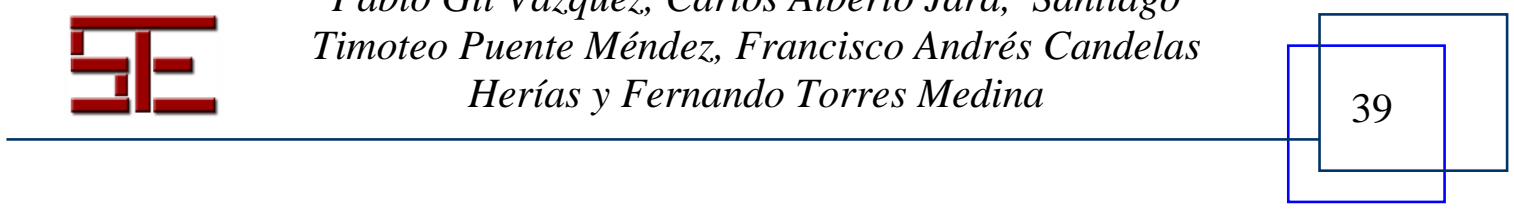




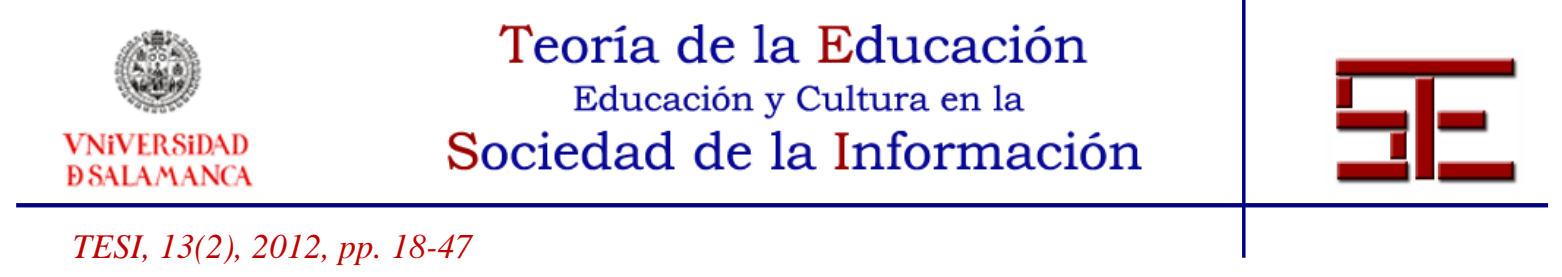

prácticos derivados tanto de la construcción e instalación del robot como del conocimiento práctico que los estudiantes han adquirido en relación a manejo de sensores, control de motores o programación. Además, estudiantes con diferentes perfiles y distintos conocimientos ingenieriles, como los que se matriculan de este tipo de cursos extracurriculares, enriquecen el aprendizaje aglutinando ideas $y$ conocimientos previos diferentes.

En un contexto educativo, donde la pedagogía de la enseñanza de la robótica es aún un campo joven, este curso de robótica, junto con el concurso con pruebas competitivas, proporcionan una oportunidad de almacenar nuevas experiencias y habilidades que complementen la formación de los estudiantes. Como se comenta en Pack (2004), las competiciones tienen una gran cantidad de factores de motivación para el estudiante y promueven las ideas y la innovación en un contexto de juego y diversión.

\section{4.- CONCLUSIONES Y PERCEPCIÓN DEL ALUMNO}

4.1.- Docencia en robótica en el ámbito extracurricular: Aprendizaje con robots de bajo coste

En los últimos años, los cursos de diseño y creación de minirrobots se han introducido con éxito en los diferentes niveles educativos desde la educación secundaria hasta la Universidad. Hay gran diversidad de plataformas y kits robóticos como se ha comentado en la introducción de este artículo (ver sección 1.1). No obstante, en los cursos extracurriculares que se imparten en la Universidad de Alicante para estudiantes universitarios, se ha escogido el modelo Skybot (ver Sección 2.1).

Pablo Gil Vázquez, Carlos Alberto Jara, Santiago

Timoteo Puente Méndez, Francisco Andrés Candelas

40 Herías y Fernando Torres Medina 


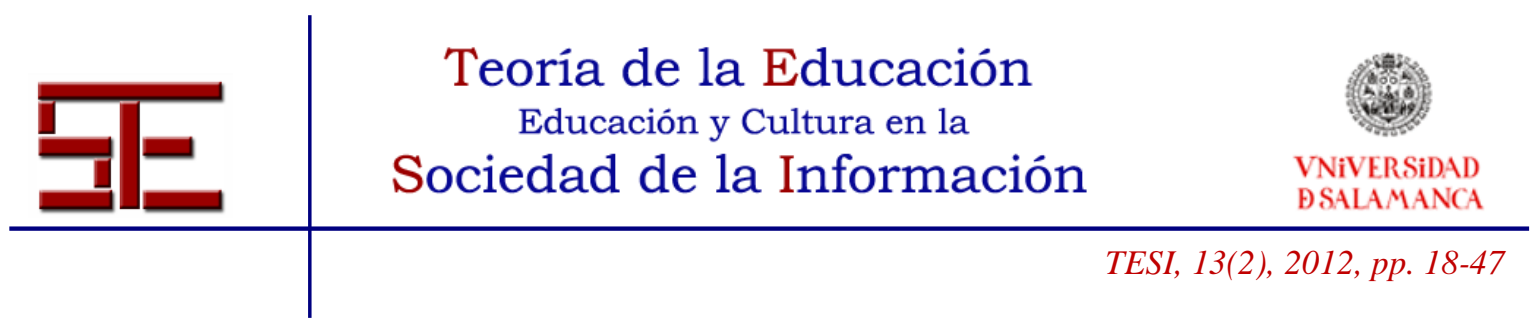

OPINIÓN SOBRE EL CURSO DE MONTAJE Y PROGRAMACIÓN DE MINIRROBOTS

\begin{tabular}{|c|c|c|c|c|c|}
\hline $\begin{array}{l}\text { Aspectos } \\
\text { Evaluables }\end{array}$ & $\begin{array}{l}\text { Tot. } \\
\text { satisfecho/ } \\
\text { de acuerdo }\end{array}$ & $\begin{array}{l}\text { Muy } \\
\text { satisfecho/de } \\
\text { acuerdo }\end{array}$ & $\begin{array}{l}\text { Satisfecho/ } \\
\text { de acuerdo }\end{array}$ & $\begin{array}{l}\text { Insatisfech } \\
\text { o/ en } \\
\text { desacuerdo }\end{array}$ & $\begin{array}{l}\text { Nada } \\
\text { satisfecho/ } \\
\text { nada de } \\
\text { acuerdo }\end{array}$ \\
\hline $\begin{array}{ll}\text { Documentación } & y \\
\text { materiales didácticos } & \end{array}$ & $20 \%$ & $20 \%$ & - & - & - \\
\hline Contenidos aprendidos & $35 \%$ & $25 \%$ & - & - & - \\
\hline Electrónica & $35 \%$ & $30 \%$ & $30 \%$ & - & - \\
\hline Microcontroladores & $70 \%$ & $25 \%$ & $5 \%$ & - & - \\
\hline Robótica & $60 \%$ & $25 \%$ & - & - & - \\
\hline Nivel de dificultad* ${ }^{*}$ & $5 \%$ & $5 \%$ & $85 \%$ & $5 \%$ & - \\
\hline $\begin{array}{l}\text { Duración y organización } \\
\text { horaria }\end{array}$ & $20 \%$ & $15 \%$ & $30 \%$ & $30 \%$ & - \\
\hline $\begin{array}{l}\text { Destrezas profesionales } \\
\text { para futuro laboral }\end{array}$ & $40 \%$ & $45 \%$ & $10 \%$ & $5 \%$ & - \\
\hline 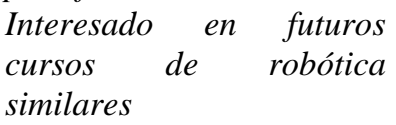 & $65 \%$ & $20 \%$ & $15 \%$ & - & - \\
\hline
\end{tabular}

Porcentaje de estudiantes

(*) El nivel de dificultad se evalúa como muy bajo, bajo, medio, alto, muy alto

Figura 8. Opinión curso de montaje y programación de mini-robots.

En referencia a estos cursos sobre robótica, los estudiantes perciben en su gran mayoría, el $40 \%$ está totalmente satisfecho y el $60 \%$ muy satisfecho del conocimiento adquirido en materias transversales como la electrónica necesaria para la construcción de un robot como Skybot. Porcentajes similares se obtienen cuando los estudiantes son preguntados por el nivel de aprendizaje que creen haber alcanzado sobre conceptos básicos propios de la robótica como efectores y actuadores, manipulación, control, sensores o planificación de movimientos y navegación, entorno, al $45 \%$ y el $50 \%$ se encuentran también totalmente satisfechos y muy satisfechos, respectivamente. De igual manera, los cursos extracurriculares de construcción y programación de robots han requerido tareas de programación y creación de algoritmos, la opinión en este sentido ha sido más diversa, pero siempre ha tenido un nivel de satisfacción medio-alto. El $25 \%$ y $35 \%$ de los estudiantes están muy satisfechos y/o totalmente satisfechos frente al $25 \%$ de los estudiantes que sólo tienen un nivel medio de satisfacción. Pero, además, en esta última edición del curso, los estudiantes han manifestado su opinión sobre si la programación horaria y la organización de los contenidos, así como la metodología empleada para impartirlos son los más adecuados para facilitar el seguimiento y aprendizaje del

Pablo Gil Vázquez, Carlos Alberto Jara, Santiago






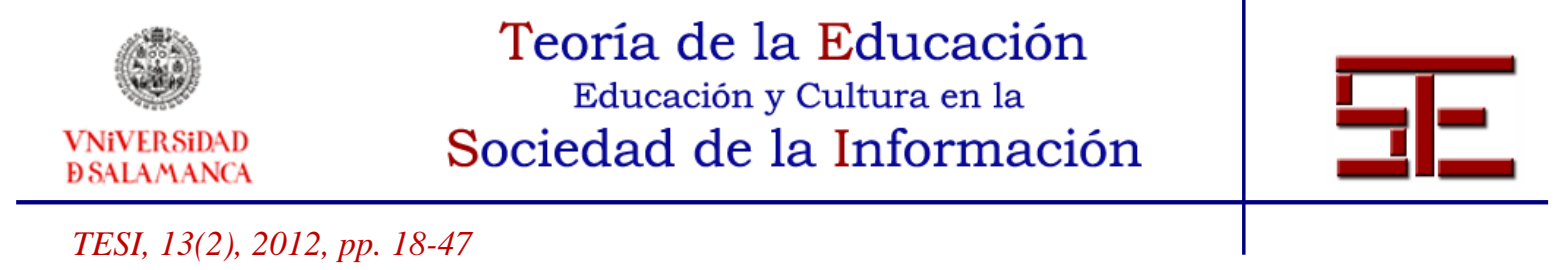

estudiante. En general, atendiendo a la información que se muestra en la Figura 8, el curso está catalogado como de fácil aprendizaje, aunque un pequeño sector de estudiantes destaca que se requieren algunas horas de más para la programación de dispositivos como el microprocesador y/o sensores. Por un lado, el 35\% de los estudiantes piensan que la duración del curso es adecuada, otro $30 \%$ restante opina que se requieren algunas horas más destinadas a cuestiones prácticas de entrenamiento para la programación de los robots. Por otro lado, el $60 \%$ de los estudiantes están satisfechos o muy satisfechos sobre los contenidos del curso, frente al $40 \%$ que no dan a conocer su opinión. En cualquier caso, el 95\% de los estudiantes encuestados están de acuerdo en manifestar que el robot Skybot es un kit robótico que permite aprender robótica y todas aquellas destrezas y contenidos interdisciplinares como la electrónica (el 70\% de los estudiantes están totalmente de acuerdo y el 25\% están de acuerdo). Además, el 85\% piensa que Skybot favorece el aprendizaje de conceptos robóticos como control de movimientos, programación de tareas o navegación (60\% está totalmente de acuerdo, $25 \%$ está de acuerdo y $15 \%$ no opina). Incluso el $95 \%$ llega a agradecer que el curso cubra técnicas de programación en tiempo real basadas en programación en microcontroladores basados en el dispositivo Arduino.

El curso extracurricular cumple los objetivos marcados como así se indica en la Figura 8 cuando los estudiantes son preguntados por las destrezas adquiridas en el curso y si éstas creen que son útiles para la incorporación al mundo laboral. En este caso, el 85\% de los estudiantes muestran una opinión muy satisfactoria o totalmente satisfactoria. Además el $100 \%$ de los estudiantes estarían interesados en volver a cursar de nuevo cursos relacionados con la robótica, similares al impartido.

\section{2.- Docencia en robótica en el ámbito de grado y post-grado: Aprendizaje con laboratorios virtuales}

Tras la realización de las prácticas, se viene pasando cada curso académico cuestionarios a los estudiantes sobre su opinión sobre el laboratorio virtual y su satisfacción con el mismo. En general, los estudiantes muestran que están totalmente o muy satisfechos con el sistema utilizado, el $82 \%$ de los estudiantes es de esta opinión. Por otro lado, la facilidad que plantea el sistema para su utilización ha sido evaluada positivamente, el $90 \%$ opinan que resulta sencilla o muy sencilla su utilización.

En lo que respecta a los conceptos aprendidos, o a la facilidad para comprender conceptos gracias a RobUALab.Ejs, el $80 \%$ opinan que se facilita el aprendizaje y la comprensión de los conceptos teóricos de la asignatura. Finalmente, se ha evaluado la

Pablo Gil Vázquez, Carlos Alberto Jara, Santiago






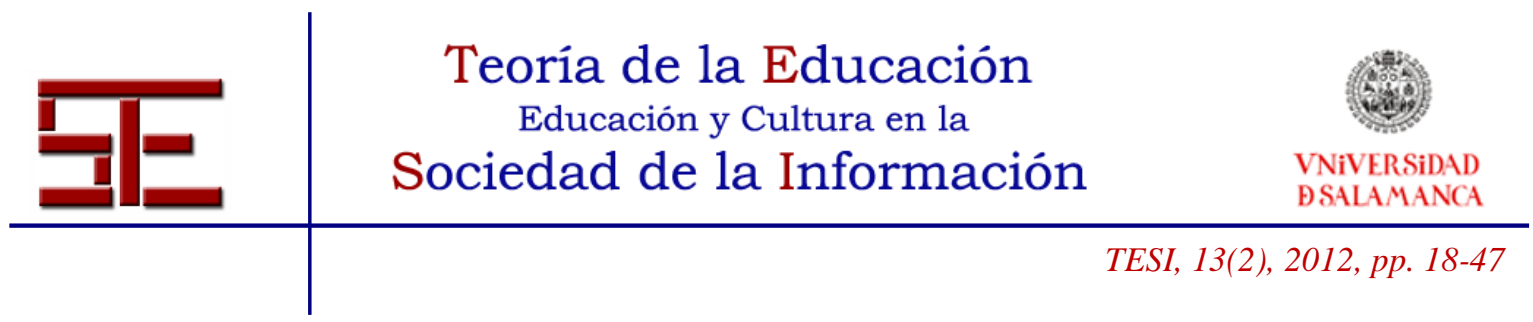

calidad del entorno, donde el $68 \%$ de los estudiantes se encuentran muy satisfechos con el mismo. Estos resultados se encuentran desarrollados en la Figura 9.

Figura 9. Opinión sobre el uso de RobUAlab.Ejs.

\begin{tabular}{|c|c|c|c|c|c|}
\hline $\begin{array}{l}\text { Aspectos } \\
\text { Evaluables }\end{array}$ & $\begin{array}{l}\text { Tot. } \\
\text { satisfecho/ } \\
\text { de acuerdo }\end{array}$ & $\begin{array}{l}\text { Muy } \\
\text { satisfecho/de } \\
\text { acuerdo }\end{array}$ & $\begin{array}{l}\text { Satisfecho/ } \\
\text { de acuerdo }\end{array}$ & $\begin{array}{l}\text { Insatisfech } \\
\text { o/ en } \\
\text { desacuerdo }\end{array}$ & $\begin{array}{l}\text { Nada } \\
\text { satisfecho/ } \\
\text { nada de } \\
\text { acuerdo }\end{array}$ \\
\hline Satisfacción del entorno. & $36 \%$ & $46 \%$ & $12 \%$ & $6 \%$ & - \\
\hline Facilidad de utilización & $46 \%$ & $44 \%$ & $10 \%$ & - & - \\
\hline $\begin{array}{l}\text { Ayuda para comprender } \\
\text { conceptos teóricos }\end{array}$ & $35 \%$ & $45 \%$ & $15 \%$ & $5 \%$ & - \\
\hline Calidad del entorno & $30 \%$ & $38 \%$ & $20 \%$ & $6 \%$ & $1 \%$ \\
\hline
\end{tabular}

Porcentaje de estudiantes

\section{5.- BIBLIOGRAFÍA}

AESSBot (1994). Competición de robots UPC: AESSBot. Extraído el 8 de mayo, 2012, de http://aess.upc.es/aessbot/.

Alcabot (2000). Competición de robots UAH: Alcabot-Hispabot. Extraído el 8 de mayo, 2012, de http://www.alcabot.com.

Alencastre, M.; Muñoz-Gómez, L. y Rudomin, I. (2003). Teleoperating robots in multiuser virtual environments. En Proceedings of the 4th Mexican International Conference on Computer Science. (pp. 314-321). México.

Assaf, D., Pfeifer, R. (2011). EmbedIT - An Open Robotic Kit for Education. En D. Obdrzalek and A. Gottscheber (Eds.), Research and Education in RoboticsEUROBOT 2011: Vol. 161. Communications in Computer and Information Science (pp. 29-39). Springer-Verlag.

AUROBot (2010). Competición de robots UA: AUROBot. Extraído el 8 de mayo, 2012, de http://www.aurova.ua.es/microbot/index.php/es/.

Balch, T., Yanco, H. A. (2002). Ten years of the AAAI mobile robot competition and exhibition: looking back and to the future. AI Magazine, 23 (1), 13-22.

Pablo Gil Vázquez, Carlos Alberto Jara, Santiago

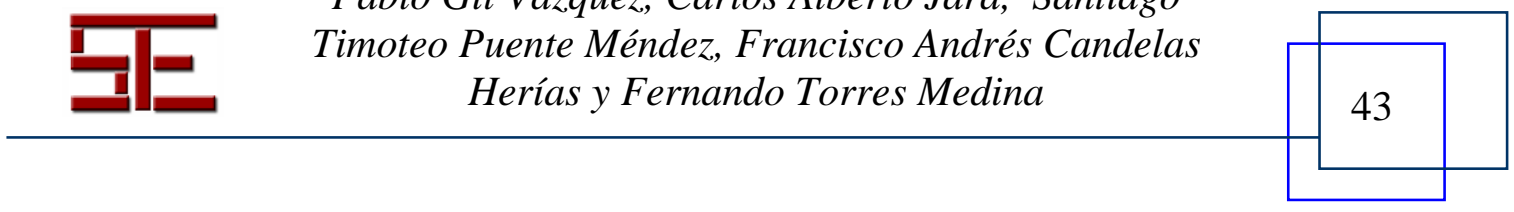




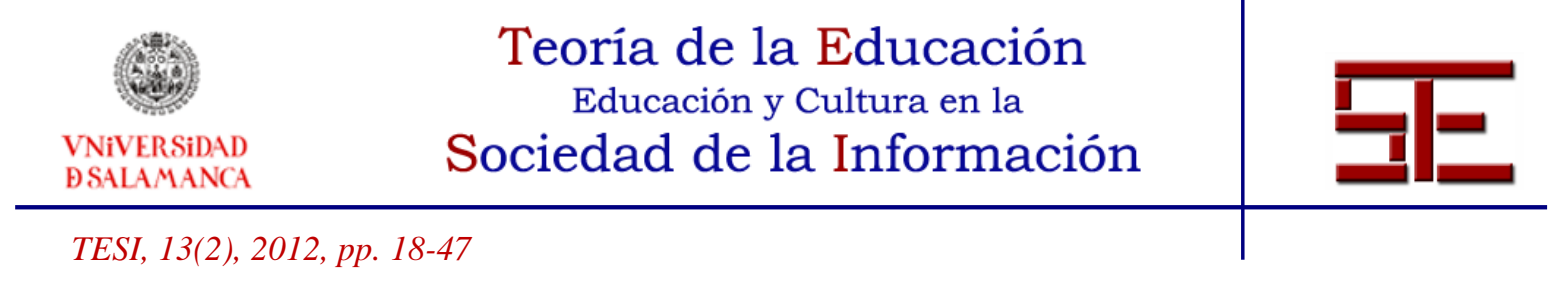

Balogh, R. (2005). A Survey of Robotic Competitions. International Journal of Advanced Robotic Systems, 2 (39), 144-160.

Biolid (2010). Robots humanoides de la empresa Robotis. Extraído el 7 de mayo, 2012, de http://www.robotis.com/xe/bioloid_en.

CampusBot (2005). Competición de robots CampusParty. Extraído el 8 de mayo, 2012, de http://www.campus-party.es/2011/campusbot.html.

Candelas, F. A., Puente, S. T., Torres, F., Ortiz, F., Gil, P., Pomares, J. (2003). A virtual laboratory for teaching robotics. International Journal of Engineering Education, 19(3), 363-370.

Candelas, F., Jara, C. \& Torres, F. (2006). Flexible virtual and remote laboratory for teaching Robotics. En Proceedings of 4th International Conference on Multimedia and Information \& Communication Technologies in Education. Sevilla.

Cañada, J. C., Sanguino, T. J. M., Alcocer, S., Borrego, A., Isidro, A., Palanco, A., Rodríguez, J. M. (2011). From Classroom to Mobile Robots Competition Arena: An Experience on Artificial Intelligence Teaching. International Journal of Engineering Education, 24(4), 1-8.

CEA-GTRob (2011). El libro blanco de la robótica en España. Investigación, tecnologías y formación. Comité Español de Automática-Gupo de Trabajo Robótica. ISBN: 978-84-615-4583-4.

CEABOT (2006). Competición de robots del Comité Español de Automática (CEA): CEABOT. Investigación, tecnologías y formación. Extraído el 7 de mayo, 2012, de http://www.ceautomatica.es/og/robotica/concurso-ceabot.

Cosma, C., Confente, M., Botturi, D., Fiorini, P. (2003). Laboratory tools for robotics and automation education. En Proceedings of IEEE International Conference on Robotics and Automation. Taiwan.

CosmoBot (2008). Competición de robots Obra Social la Caixa: CosmoBot. Extraído el 8 de mayo, 2012, de http://www.roboticspot.com/cosmobot/index.php.

Couceiro, M. S., Figueiredo, C. M., Luz, J. M. A. \& Ferreira, M. F. (2011). A Low-Cost Educational Platform for Swarm Robotics. International Journal of Robotics, Education and Art, 2 (1), 1-15.

Cybertech (2001). Competición de robots UPM. Extraído el 7 de mayo, 2012, de http://reset.etsii.upm.es/es/projects/cybertech/.

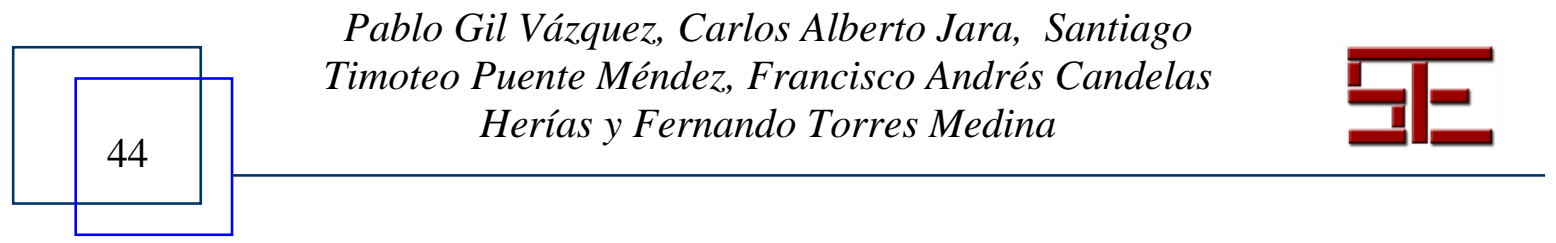




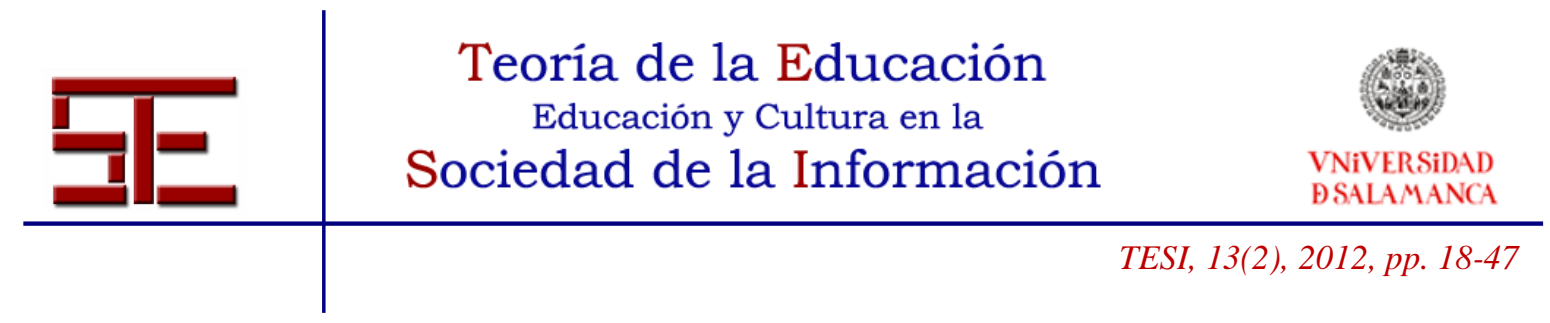

González-Fierro, M., Jardón, A., Martínez de la Casa, S., Stoelen, M. F., Victores, J. G., Balaguer, C. (2010). Educational initiatives related with the CEABOT contest. En Proceedings of the International Conference on Simulation, Modeling and Programming for Autonomous Robots. (pp. 649-658).

Darwin-op (2011). Robots humanoide DARwIn-op de la empresa Robotis. Extraído el 7 de mayo, 2012, de http://www.robotsource.org/xe/.

Dormido, S. (2004). Control learning: present and future. Annual Reviews in Control, 28, 115-136.

Esquembre, F. (2004). Easy Java Simulations: A software tool to create scientific simulations in Java. Computer Physics Communications, 156 (2), 199-204.

Hernando, M., Galán, R., Navarro, I., Rodríguez-Losada, D. (2011). Ten Years of Cybertech: The Educational Benefits of Bullfighting Robotics. IEEE Transactions on Education, 54 (4), 569-575.

Jara, C. A., Candelas, F. A., Gil, P., Torres, F., Esquembre, F. \& Dormido, S. (2011). EJS+EjsRL: an interactive tool for Robotics simulation, Computer Vision processing and remote operation. Application to virtual and remote laboratories. Robotics and Autonomous System, 59 (6), 389-401.

Jara, C. A., Candelas, F. A., Puente, S. T. \& Torres, F. (2011). Hands-on Experiences of undergraduate students in automatics and robotics using a virtual and remote laboratory. Computer \& Education, 57 (4), 2451-2461.

Kitano, H., Asada, M., Noda, I. \& Matsubara, H. (1998). RoboCup: Robot World Cup. IEEE Robotics and Automation Magazine, 5 (3), 30-36.

K-Team (1999). K-Team Mobile Robotics. Extraído el 7 de mayo, 2012, de http://www.k-team.com/.

Lego (1998). LEGO Mindstorms. Extraído el 7 de mayo, 2012, de http://mindstorms.lego.com/.

- (1999). Competición de robots: LEGO league. Extraído el 7 de mayo, 2012, de http//www.firstlegoleague.org/.

Marin, R., Sanz, P. J., Nebot, P. \& Wirz, R. (2005). A multimodal interface to control a robot arm via the web: A case study on remote programming. IEEE Transactions on Industrial Electronics, 52 (6), 1506-1520.

Pablo Gil Vázquez, Carlos Alberto Jara, Santiago Timoteo Puente Méndez, Francisco Andrés Candelas Herías y Fernando Torres Medina 


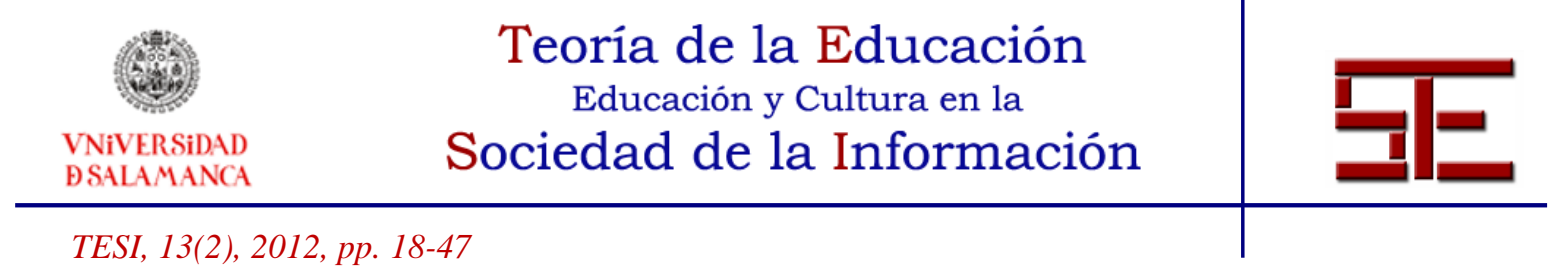

Murphy, R. R. (2001). Competing for a robotics competition. IEEE Robotics \& Automation Magazine, 8 (2), 44-55.

Nourbakhsh, I. R., Crowley, K., Bhave, A., Hamner, E., Hsiu, T., Perez-Bergquist, A., Richards, S. \& Wilkinson, K. (2005). The Robotic Autonomy Mobile Robotics Course: Robot Design, Curriculum Design and Educational Assessment. Autonomous Robots, 18, 103-127.

Pack, D. J., Avanzato, R., Ahlgren, D. J. \& Verner, I.M. (2004). Fire-Fighting Mobile Robotics and Interdisciplinary Design-Comparative Perspectives. IEEE Transactions on Education, 47 (1), 369-376.

Puente, S., Torres, F., Candelas, F. \& Ortiz, F. (2000). Remote Robot Execution through WWW Simulation. En Proceedings of the 15th International Conference on Pattern Recognition. Piscataway (USA).

Puente, S. T., Candelas, F. A., Gil, P., Perea, I., García, G.J., Jara, C.A. \& Corrales, J. A. (2012). An experience on Mechatronics teaching on undergraduate students by means of the Skybot platform: from classroom to robot competition. International Journal of Robotics, Education and Art.

Robocup (1996). Competición de robots: ROBOCUP. Extraído el 8 de mayo, 2012, de http://www.robocup.org/.

Robolid (2003). Competición de robots UV. Extraído el 8 de mayo, 2012, de http://www.robolid.net/.

Robonova (2005). Robots humanoides de la empresa Robotics HiTec. Extraído el 7 de mayo, 2012, de http://www.robonova.de/store/home.php.

Roboparty (2012). Competición de robots Universidad do Minho en Portugal. Extraído el 8 de mayo, 2012, de http://www.roboparty.org/.

Robótica (2001). Competición de robots de la Sociedad de Robótica Portuguesa (SPR). Extraído el 8 de mayo, 2012, de http://www.spr.ua.pt/fnr/.

Safaric, R., Truntic, M., Hercog, D. (2005). Control and robotics remote laboratory for engineering education. Interational Journal of Online Engineering (iJOE), 1 (1).

Skybot (2008). Robot Skybot. Extraído el 7 de mayo, 2012, de http://www.iearobotics.com/wiki/index.php?title=Skybot.

Pablo Gil Vázquez, Carlos Alberto Jara, Santiago

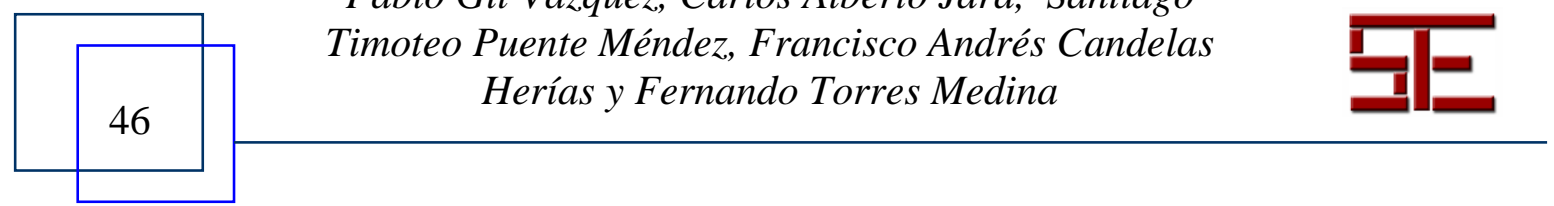




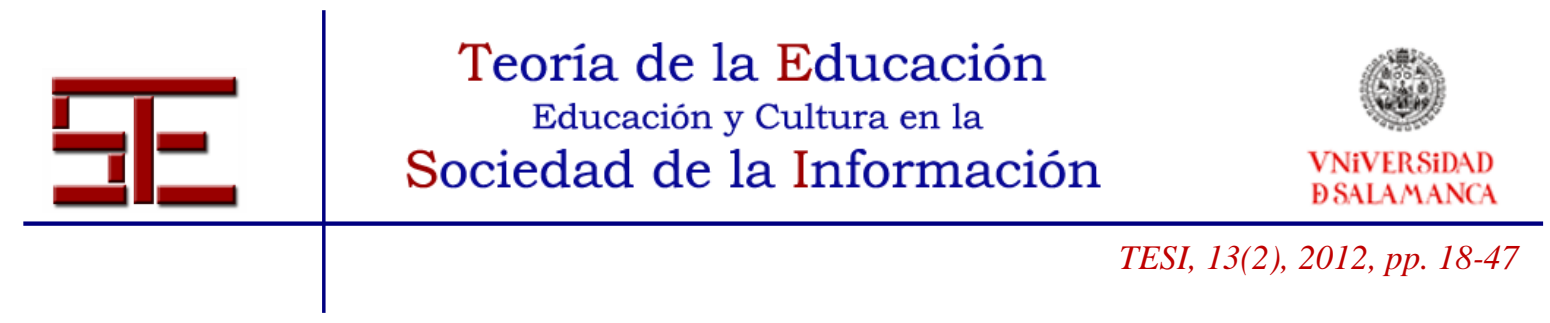

Torres, F., Candelas, F. A., Puente, S. T., Pomares, J., Gil, P. \& Ortiz, F. G. (2006). Experiences with Virtual Environment and Remote Laboratory for Teaching and Learning Robotics at University of Alicante. International Journal of Engineering Education, 22 (4), 766-776.

Torres, F., Puente, S., Damas, I., Puerto, C. y Candelas, F. (1999). ASTRO: Aprendizaje mediante Simulación y Teleoperación de Robots. En Actas de las XX Jornadas de Automática. Salamanca.

Tzafestas, C. S., Palaiologou, N. Alifragis, M. (2006). Virtual and remote robotic laboratory: comparative experimental evaluation. IEEE Transactions on Education, 49(3), 360-369.

Vargas, H., Sánchez, J., Jara, C. A., Candelas, F. A. Reinoso, O. \& Díez, J. L. (2010). Docencia en Automática: Aplicación de las TIC a la realización de actividades prácticas a través de Internet. Revista Iberoamericana de Automática $e$ Informática Industrial, 7 (1), 35-45.

Vavassori, F. B. (2012). Exploring the educational potential of robotics in schools: A systematic review. Computers \& Education, 58 (3), 978-988.

Vex (2007). VEX Robotics Design System. Extraído el 7 de mayo, 2012, de http://www.vexrobotics.com/.

Para citar el presente artículo puede utilizar la siguiente referencia:

Gil Vázquez, P., Jara, C. A., Puente Méndez, S. T., Candelas Herías, F. A. y Torres Medina, F. (2012). Recursos y herramientas didácticas para el aprendizaje de la robótica. Revista Teoría de la Educación: Educación y Cultura en la Sociedad de la Información. 13(2), 18-47 [Fecha de consulta: dd/mm/aaaa].

http://campus.usal.es/ revistas_trabajo/index.php/revistatesi/article/view/8998/9243

Pablo Gil Vázquez, Carlos Alberto Jara, Santiago

Timoteo Puente Méndez, Francisco Andrés Candelas Herías y Fernando Torres Medina 